\title{
Closed-form and finite difference solutions to a population balance model of grinding mills
}

\author{
Raimund Bürger (buerger@mathematik.uni-stuttgart.de) \\ Institute of Applied Analysis and Numerical Simulation, University of Stuttgart, Pfaffenwaldring 57, D-70569 \\ Stuttgart, Germany
}

Kenneth H. Karlsen (kennethk@math.uio.no)

Centre of Mathematics for Applications (CMA), University of Oslo, P.O. Box 1053, Blindern, N-0316 Oslo, Norway

John D. Towers (jtowers@cts.com)

MiraCosta College, 3333 Manchester Avenue, Cardiff-by-the-Sea, CA 92007-1516, USA

October 7, 2004

\begin{abstract}
The wear of steel balls in continuously operated grinding mills, used in mineral processing to comminute metalliferous rocks, can be described by a simple population balance model. This model gives rise to a scalar transport equation with a singular source term for the number density of balls as a function of size and time. Exact solutions to this equation are determined under the assumption of a simple power-law type wear law. It is shown that a particular term proposed in the engineering literature that describes the removal of used balls from the mill leads to negative solutions (Model 1). An alternative, more realistic term for the sieve action, which admits nonnegative solutions only, is introduced (Model 2). A working first-order finite difference scheme for Model 2 and a second-order TVD variant are introduced and applied for numerical simulations along with an error study. A weak solution concept for Model 2 is proposed, uniqueness of weak solutions is shown and convergence of the first-order scheme to a weak solution is established. These results hold for a general class of wear laws, not just power-law type.
\end{abstract}

Keywords: population balance model, linear transport equation, finite-difference scheme, TVD scheme, numerical simulation, uniqueness, existence

\section{Introduction}

Population balance models were formulated for chemical engineering purposes in the celebrated paper by Hulburt and Katz [1]. Currently, these models are widely used to describe and control a wide range of particulate processes including comminution, crystallization, granulation, flocculation, combustion, and polymerization [2]. In general, these models refer to dispersed systems that include both extensional and internal space-type coordinates, and in which the dispersed particles (or droplets) that form a population interact with each other, which includes collision, breakage, coalescence, and agglomeration processes. Moreover, these models include 'birth' and 'death' processes, which take place, for examples, when particles are added to or sieved out from the population. In general, population balance models are governed by integro-differential equations for the distribution density of the particles of the population.

The early engineering literature on population balance modeling in various fields also includes, besides [1], the papers by Randolph and Larson [3] (who seem to be the first

(c) 2004 Kluwer Academic Publishers. Printed in the Netherlands. 
to use the term 'population balance'), Valentas, Bilous and Amundson [4], Valentas and Amundson [5], Frederickson, Ramkrishna and Tsuchiya [6], and Sastry and Fuerstenau [7]. The current standard reference on the theory, simulation, and applications of these models in engineering is the book by Ramkrishna [8]. Recent works on general population balance modeling that do not refer to a specific dispersed system include the papers by Verkoeijen et al. [2] and McCoy [9], while a thorough review of the state of the art in numerical techniques along with an exhaustive list of references is offered in the very recent paper by Attarakih, Bart and Faqir [10].

In this paper, we consider a very simple population balance model for the wear of steel balls that are used in grinding mills to aid the crushing of minerals [11, Chapter 20]. These balls are assumed to be made of the same material, and the only property in which they differ is the size $x$, which is the only coordinate that appears (besides time) in our population balance model. Since the steel consumption due to ball wear is a decisive cost factor in the operation of a ball mill, there is considerable engineering interest in identifying the appropriate wear law, in mathematical models predicting the ball size distribution, and in implementing them, for example, to optimize the make-up ball charge in a grinding mill (see [12-15]). We also mention that variants of the model presented herein that include impact energy distribution and cylindrical grinding media (rather than balls) were proposed by Datta and Rajamani [16] and Yildirim and Austin [17], respectively.

We are interested in determining the ball size distribution as a function of time. The sought quantity is the number density $u=u(x, t)$ as a function of ball size $x$ and time $t$, where by the 'size' of a ball we always mean its diameter. Thus, the number of balls having size between $x_{a}$ and $x_{b}$ at time $t$ is given by

$$
n\left(x_{a}, x_{b}, t\right)=\int_{x_{a}}^{x_{b}} u(\xi, t) d \xi
$$

We assume that the rate of decrease of the diameter of a ball of size $x$ is given by a function $g=g(x)$. A frequently used equation is

$$
g(x)=\alpha\left(x / x_{0}\right)^{\beta}, \quad 0 \leq \beta \leq 1, \quad \alpha<0,
$$

where $x_{0}$ is a reference size and the parameter $\alpha$ describes the rate of size decrease of a single ball under milling operation, and therefore has the dimension of a velocity. Actually, the parameter $\alpha$ measures the quality of the material the steel balls are manufactured of; high-quality balls are made of relatively hard material and will lead to a low absolute value of $\alpha$, while low-quality balls will be ground more rapidly and imply large absolute values of $\alpha$. Typical values of $\alpha$ for steel balls of one to four inches in diameter range from -0.05 to -0.01 millimeters per hour, see $[13,14]$.

In the mineral processing literature, the dimensionless parameter $\beta$ is sometimes referred to as 'Austin-Klimpel parameter' [18]. The value $\beta=0$ corresponds to the Bond wear law $[19,20]$, stating that the wear rate of a ball is proportional to its surface area, while $\beta=1$ is the Davis wear law [21] postulating that the wear rate is proportional to the ball volume. The former case corresponds to an abrasive mechanism of ball size reduction and the latter to an impactive one. Real ball mills usually exhibit a combination of both mechanisms, which suggests admitting the whole parameter interval $\beta \in[0,1]$. Furthermore, we assume that the grinding process is controlled by the total number feed rate $Q_{\mathrm{F}}(t)$ of new balls at the inlet of the mill, expressed as number of balls per time unit, and by the relative number frequency 
$m_{\mathrm{F}}(x)$ of balls of size $x$ at the inflow. The dimension of $m_{\mathrm{F}}(x)$ is the inverse of size, since integrating $m_{\mathrm{F}}(x)$ over a size interval $x \in[a, b]$ yields the relative frequency of balls having size between $a$ and $b$. This relative frequency is a dimensionless number between zero and one.

The new balls are assumed to have the $p$ discrete sizes $x^{1}>x^{2}>\cdots>x^{p}$. The discharge of balls is controlled by a sink term $q_{\mathrm{D}}=q_{\mathrm{D}}(x, t, u)$, which has the unit of number of balls per time unit and size unit. We will discuss two alternative specific choices for this term later. Under the present assumptions and letting $\delta(\cdot)$ denote the usual Dirac $\delta$ function, we can state the governing equation of the population balance model as

$$
\frac{\partial u}{\partial t}+\frac{\partial}{\partial x}(g(x) u(x, t))=Q_{\mathrm{F}}(t) \sum_{k=1}^{p} m_{\mathrm{F}}^{k} \delta\left(x-x^{k}\right)-q_{\mathrm{D}}(x, t, u),
$$

where $m_{\mathrm{F}}^{k}:=m_{\mathrm{F}}\left(x^{k}\right)$, and we assume that an initial particle size distribution is given as

$$
u(x, 0)=u_{0}(x), \quad x \in\left[0, x^{p}\right] .
$$

Equation (1.2) can be written in conservative form as

$$
\frac{\partial u}{\partial t}+\frac{\partial}{\partial x}\left(g(x) u(x, t)-Q_{\mathrm{F}}(t) \sum_{k=1}^{p} m_{\mathrm{F}}^{k} H\left(x-x^{k}\right)+Q_{\mathrm{D}}(t) H\left(x-x_{\min }\right)\right)=-q_{\mathrm{D}}(x, t, u),
$$

where $H(\cdot)$ is the Heaviside function. Equation (1.4) is a first-order transport equation with a discontinuous coefficient and a source term. We consider (1.4) on the domain

$$
\Pi_{T}:=\{(x, t): 0<x<X, 0<t<T\}, \quad \bar{\Pi}_{T}:=\Pi_{T} \cup \partial \Pi_{T},
$$

where $X$ is a maximum ball size with $X>x^{1}$, where $x^{1}$ is the size of the largest feed balls. Recall that balls are always ground to smaller size, so $u(X, T)=0$ for $t \in(0, T]$ arises as a natural boundary condition for (1.4).

The remainder of this paper is organized as follows. In Section 2 we first derive a closedform solution with $g(x)$ given by $(1.1)$ and the term $q_{\mathrm{D}}(x, t, u)$ used in $[13,14]$, denoted by 'Model 1'. It turns out that this choice implies negative number densities in certain cases. We propose an alternative discharge term $q_{\mathrm{D}}(x, t, u)$, which models the sieve, through which the used balls are discharged, more realistically. This alternative model is referred to as 'Model 2'. Exact solutions to Model 2 combined with the wear law (1.1) are determined and visualized for five different combinations of the parameters $\alpha$ and $\beta$. We illustrate that solutions of Model 2 are nonnegative but in general unbounded.

In general it may become complicated to derive closed-form solutions. Moreover, even if "representation formulas" for the solutions can be found, they typically involve integrals that must be evaluated numerically. Hence, numerical schemes to produce approximate solutions may be desirable. The design of such schemes is the topic of Section 3. In Subsection 3.1 we introduce a simple first-order upwind difference scheme, while in Subsection 3.2 we present a novel second-order version of the scheme. The novelty of this algorithm lies in our choice of a regularity condition to enforce. Our motivation here is that the actual solution of a conservation law with discontinuous coefficients is not itself Total Variation Diminshing (TVD), whereas its flux does have this regularity property. We refer to this property as fluxTVD. Thus, in our second-order scheme, we apply flux limiters that enforce the flux-TVD 
property, rather than the standard TVD property for nonlinear conservation laws. Section 4 presents numerical simulations and an error study for Model 2 for three values of $\beta$, using both the first-order and the second-order schemes. Both schemes accurately approximate the stationary jumps in the solution located at $x=x^{1}, \ldots, x^{p}$ at any discretization. Away from these locations, jumps of the solution are smeared out by artificial diffusion, where the degree of smearing depends on the spatial accuracy and order of the method. This is, of course, the well-known behaviour of standard schemes for conservation laws [22].

The numerical examples and recorded errors illustrate the gain in accuracy attained by using the second-order variant of the scheme for transient solutions. On the other hand, we see that due to the presence of stationary discontinuities, large time, near steady state solutions are approximated to at most first order (in the $L^{1}$ sense) by both schemes.

On the rigorous mathematical side, we have in this paper included two appendices on the analysis of Model 2. As candidate solutions are in general discontinuous, they need to be defined as weak solutions. The unboundedness of solutions requires that we also introduce a particular weighted $L^{1}$ norm. The solution concept along with a statement of the assumptions is presented in Appendix A, which also includes a uniqueness theorem for weak solutions. As a theoretical justification of our numerical scheme, we prove in Appendix B that the first-order version of our scheme converges as the discretization parameters tend to zero to the unique weak solution of Model 2. A feature of our analysis is that we establish compactness of the numerical flux, not the numerical solution itself, by bounding its total variation. The specific design of the second order scheme in Subsection 3.2 is motivated by this feature.

\section{The ball mill models and closed-form solutions}

\subsection{Model 1}

Models 1 and 2 differ in the description of the discharge mechanism. According to Menacho and Concha $[13,14]$, there exists a size $x_{\min }$ at which the balls leave the mill. Balls of this size are extracted at the prescribed discharge number rate $Q_{\mathrm{D}}(t) \geq 0$, such that

$$
q_{\mathrm{D}}(x, t, u)=Q_{\mathrm{D}}(t) \delta\left(x-x_{\min }\right) .
$$

We now determine an exact solution to Model 1, that is, to (1.2) (or (1.4)) with the initial condition (1.3) and (2.1). Using the method of characteristics for the homogeneous problem

$$
\frac{\partial u}{\partial t}+\frac{\partial}{\partial x}(g(x) u)=0, \quad u(x, 0)=u_{0}(x),
$$

we obtain the homogeneous solution

$$
u_{\mathrm{H}}(x, t)=\frac{g(\xi(x, t))}{g(x)} u_{0}(\xi(x, t))
$$

where $\xi(x, t)$ is the location where the backward characteristic through $(x, t)$ intersects the $x$-axis. An explicit formula for $\xi(x, t)$ when $g(x)$ is given by (1.1) is

$$
\xi(x, t)= \begin{cases}x \exp \left(-\alpha t / x_{0}\right) & \text { for } \beta=1, \\ {\left[x^{1-\beta}-\frac{\alpha}{x_{0}^{\beta}}(1-\beta) t\right]^{\frac{1}{1-\beta}}} & \text { for } \beta<1 .\end{cases}
$$


Note that $\xi(x, 0)=x$, and $\xi(x, t)>x$ for $t>0$, since the characteristics flow from right to left in the $x$ - $t$ plane. For the inhomogeneous problem with right-hand side $R(x, t)$, i.e.

$$
\frac{\partial u}{\partial t}+\frac{\partial}{\partial x}(g(x) u)=R(x, t), \quad u(x, 0)=u_{0}(x),
$$

an application of Duhamel's principle [23] yields

$$
u(x, t)=u_{\mathrm{H}}(x, t)+\int_{0}^{t} \frac{g(\xi(x, t-s))}{g(x)} R(\xi(x, t-s), s) d s .
$$

When $R=R(x)$, it is possible to simplify the inhomogeneous portion of the solution by making a change of variables. We let $\sigma=\xi(x, t-s)$ and after some algebra, discover that $d s=d \sigma / g(\sigma)$. The solution then reduces to

$$
u(x, t)=u_{\mathrm{H}}(x, t)-\frac{1}{g(x)} \int_{x}^{\xi(x, t)} R(\sigma) d \sigma=u_{\mathrm{H}}(x, t)+\frac{1}{g(x)} \int_{\xi(x, t)}^{x} R(\sigma) d \sigma .
$$

When the right-hand side $R$ takes the following specific form:

$$
R(x)=Q_{\mathrm{F}} \sum_{k=1}^{p} m_{\mathrm{F}}^{k} \delta\left(x-x^{k}\right)-Q_{\mathrm{D}} \delta\left(x-x_{\min }\right),
$$

which corresponds to choosing constant feed and discharge rates $Q_{\mathrm{F}}$ and $Q_{\mathrm{D}}$ in Model 1, the solution finally turns out to be

$$
u(x, t)=u_{\mathrm{H}}(x, t)+\frac{1}{g(x)}\left[Q_{\mathrm{F}} \sum_{k=1}^{p} m_{\mathrm{F}}^{k} \chi_{[x, \xi(x, t)]}\left(x^{k}\right)-Q_{\mathrm{D}} \chi_{[x, \xi(x, t)]}\left(x_{\min }\right)\right],
$$

where

$$
\chi_{[a, b]}(x):= \begin{cases}1 & \text { if } x \in[a, b] \\ 0 & \text { otherwise }\end{cases}
$$

denotes the characteristic function of an interval $[a, b]$. Note that (2.3) defines a function that is in general discontinuous across the straight lines $x=x^{1}, \ldots, x^{p}, x_{\min }$ and the curves $t \mapsto\left(\xi\left(x^{k}, t\right), t\right), k=1, \ldots, p$ and $t \mapsto\left(\xi\left(x_{\min }, t\right), t\right)$. Consequently, (2.3) does not satisfy (1.4) in a pointwise sense; rather, this function is a weak solution. The appropriate mathematical concept of weak solutions in detailed in Appendix A.

Model 1 has successfully solved some engineering problems [13, 14], but it has a shortcoming that becomes apparent in (2.3). Namely, the discharge rate of balls at a given point of time does not involve the solution $u$. To illustrate the consequence of this property, assume that we start from an empty ball mill $\left(u_{0} \equiv 0\right)$ and feed no new balls into it $\left(Q_{\mathrm{F}}=0\right)$, but let $Q_{\mathrm{D}}>0$, i.e. one attempts to extract balls at a given rate. A sound mathematical model should then predict a zero discharge flux of balls, and that the ball mill remains empty, i.e. $n\left(x_{a}, x_{b}, t\right)=0$ for all $0 \leq x_{a}<x_{b} \leq X$. However, (2.3) implies $u(x, t)=-Q_{\mathrm{D}} \chi_{[x, \xi(x, t)]}\left(x_{\min }\right) / g(x)$ and in particular $n\left(x_{a}, x_{b}, t\right)<0$ if $\left(x_{a}, x_{b}\right) \cap(\xi(x, t), x) \neq \varnothing$. Negative densities and numbers are, however, undesirable and call for improvement of Model 1. 


\subsection{Model 2}

Model 1 can be improved if we model the discharge mechanism in a different way. Instead of assuming that there is a prescribed discharge rate of balls of a determined size, which seems quite unrealistic and difficult to implement, we consider that balls of size $x \leq x_{\mathrm{s}}$ fall automatically at all times through the discharge sieve of meshwidth $x_{\mathrm{s}}$. (For realizations of such discharge openings in real grinding mills we refer to [11, pp. 20-31 ff.].) This selection effect can be considered if we skip the discharge term from (3.1) and rewrite the equation in integral form. Then we have, first without sieve and considering $x_{a}, x_{b} \neq x^{1}, \ldots, x^{p}$,

$$
\begin{aligned}
n\left(x_{a}, x_{b}, t+\Delta t\right)-n\left(x_{a}, x_{b}, t\right)= & -\int_{t}^{t+\Delta t}\left(g\left(x_{b}\right) u\left(x_{b}, \tau\right)-g\left(x_{a}\right) u\left(x_{a}, \tau\right)\right) d \tau \\
& +\sum_{k=1, x_{a}<x^{k}<x_{b}}^{p} m_{\mathrm{F}}^{k} \int_{t}^{t+\Delta t} Q_{\mathrm{F}}(\tau) d \tau
\end{aligned}
$$

The sieve is not present if $x_{a}>x_{\mathrm{s}}$. Moreover, we assume that the action of the sieve is also associated with a time-dependent control function $Q_{\mathrm{S}}(t)$. The total amount of balls of size between $x_{a}$ and $x_{b}$ that are sieved out in the time interval $[t, t+\Delta t]$ is given by

$$
n_{\mathrm{S}}\left(x_{a}, x_{b}, t, t+\Delta t\right)=\int_{t}^{t+\Delta t} \int_{x_{a}}^{x_{b}} Q_{\mathrm{S}}(\tau) \chi_{\left[0, x_{\mathrm{s}}\right]}(\xi) u(\xi, \tau) d \xi d \tau
$$

This quantity has to be subtracted from the right-hand side of (2.4) to appropriately model the sieve action. Thus, the alternative discharge term is

$$
q_{\mathrm{D}}(x, t, u)=m_{\mathrm{F}}^{k} \delta\left(x-x^{k}\right) Q_{\mathrm{S}}(t) \chi_{\left[0, x_{\mathrm{s}}\right]}(x) u(x, t) .
$$

Observe that the equation (1.2) in conjunction with (2.5) is still linear, but slightly more involved than the equation for Model 1 since the sink term now depends on the solution $u$. For easy reference, we rewrite the governing equation for Model 2 as

$$
\frac{\partial u}{\partial t}+\frac{\partial}{\partial x}\left(g(x) u(x, t)-Q_{\mathrm{F}}(t) \sum_{k=1}^{p} m_{\mathrm{F}}^{k} H\left(x-x^{k}\right)\right)=-Q_{\mathrm{S}}(t) \chi_{\left[0, x_{\mathrm{S}}\right]}(x) u(x, t) .
$$

We will limit the further analysis to that case. We should expect that unlike Model 1, Model 2 produces nonnegative solutions only. This will be established in Section 6 by the analysis of a difference scheme.

\subsection{Exact SOlution of Model 2}

To simplify the analysis, we assume that the coefficients are time-independent, and we simplify the notation for the sieve term by setting

$$
b(x):=-Q_{\mathrm{S}} \chi_{\left[0, x_{\mathrm{s}}\right]}(x) .
$$

Using the method of characteristics for the resulting homogeneous problem

$$
\frac{\partial u}{\partial t}+\frac{\partial}{\partial x}(g(x) u)-b(x) u=0, \quad u(x, 0)=u_{0}(x),
$$


we obtain the homogeneous solution

$$
u_{\mathrm{H}}(x, t)=\frac{g(\xi)}{g(x)} u_{0}(\xi) \exp \left(\int_{\xi}^{x} \frac{b(s)}{g(s)} d s\right)
$$

where $\xi(x, t)$ is given by (2.2) if $g(x)$ is defined by (1.1).

For the inhomogeneous problem with a time-independent right-hand side $R(x)$, i.e.,

$$
\frac{\partial u}{\partial t}+\frac{\partial}{\partial x}(g(x) u)-b(x) u=R(x), \quad u(x, 0)=u_{0}(x),
$$

we apply Duhamel's principle as we did for Model 1, this time obtaining

$$
u(x, t)=u_{\mathrm{H}}(x, t)+\int_{0}^{t} w(x, t, \tau) d \tau,
$$

where

$$
w(x, t, \tau):=\frac{g(\hat{\xi})}{g(x)} R(\hat{\xi}) \exp \left(\int_{\hat{\xi}}^{x} \frac{b(s)}{g(s)} d s\right), \quad \hat{\xi}:=\xi(x, t-\tau) .
$$

With the change of variables $\rho=\hat{\xi}=\xi(x, t-\tau), d \tau=d \rho / g(\rho)$, the solution then reduces to

$$
u(x, t)=u_{\mathrm{H}}(x, t)-\frac{1}{g(x)} \int_{x}^{\xi(x, t)} R(\rho) \exp \left(\int_{\rho}^{x} \frac{b(s)}{g(s)} d s\right) d \rho .
$$

For Model 2 with constant feed rates, the right-hand side $R$ consists of a sum of source terms:

$$
R(x)=Q_{\mathrm{F}} \sum_{k=1}^{p} m_{\mathrm{F}}^{k} \delta\left(x-x^{k}\right)
$$

The presence of the $\delta$ functions allows us to simplify the exact solution, since

$$
\begin{aligned}
\int_{x}^{\xi(x, t)} R(\rho) \exp \left(\int_{\rho}^{x} \frac{b(s)}{g(s)} d s\right) d \rho & =\int_{x}^{\xi(x, t)} Q_{\mathrm{F}} \sum_{k=1}^{p} m_{\mathrm{F}}^{k} \delta\left(\rho-x^{k}\right) \exp \left(\int_{\rho}^{x} \frac{b(s)}{g(s)} d s\right) d \rho \\
& =Q_{\mathrm{F}} \sum_{k=1}^{p} m_{\mathrm{F}}^{k} \chi_{[x, \xi(x, t)]}\left(x^{k}\right) \exp \left(\int_{x^{k}}^{x} \frac{b(s)}{g(s)} d s\right) .
\end{aligned}
$$

Substituting this expression into (2.9), we obtain

$$
u(x, t)=u_{\mathrm{H}}(x, t)-\frac{Q_{\mathrm{F}}}{g(x)} Q_{\mathrm{F}} \sum_{k=1}^{p} m_{\mathrm{F}}^{k} \chi_{[x, \xi(x, t)]}\left(x^{k}\right) \exp \left(\int_{x^{k}}^{x} \frac{b(s)}{g(s)} d s\right) .
$$

Using (2.7), it is possible to work out explicit formulas for the integrals of the form

$$
\mathcal{I}\left(z_{1}, z_{2}\right):=\int_{z_{1}}^{z_{2}} \frac{b(s)}{g(s)} d s
$$

that appear in both the homogeneous and inhomogeneous portions of the solution. Specifically for the wear law (1.1), we get

$$
\mathcal{I}\left(z_{1}, z_{2}\right)=-\frac{Q_{\mathrm{S}} x_{0}^{\beta}}{\alpha} \begin{cases}\ln \left(\min \left\{x_{\mathrm{s}}, z_{2}\right\}\right)-\ln \left(\min \left\{x_{\mathrm{s}}, z_{1}\right\}\right) & \text { for } \beta=1, \\ \frac{1}{1-\beta}\left(\left(\min \left\{x_{\mathrm{s}}, z_{2}\right\}\right)^{1-\beta}-\left(\min \left\{x_{\mathrm{s}}, z_{1}\right\}\right)^{1-\beta}\right) & \text { for } \beta \neq 1 .\end{cases}
$$



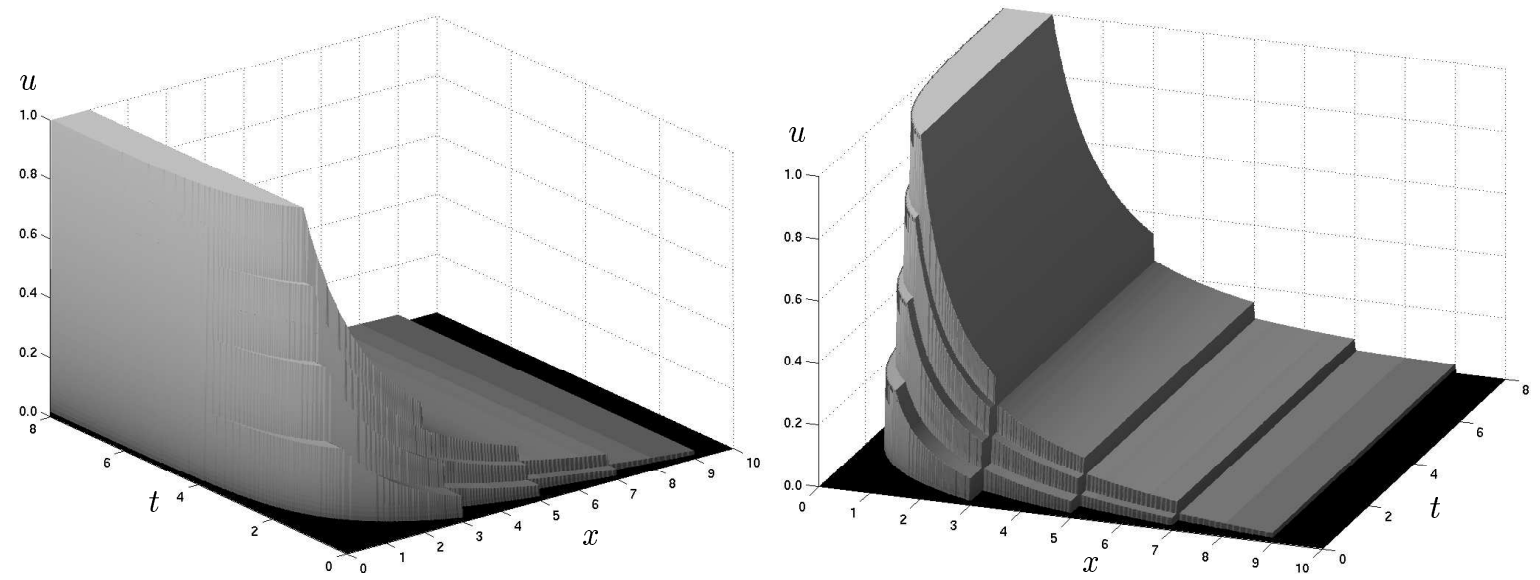

Figure 1. Exact solution of Model 2 with $\beta=0$.

It is evident from this formula that $\mathcal{I}\left(z_{1}, z_{2}\right)=0$ if both $z_{1} \geq x_{\mathrm{s}}$ and $z_{2} \geq x_{\mathrm{s}}$, indicating that the sieve has no affect on the solution for $x>x_{\mathrm{s}}$. For easy reference, we collect in one place the exact solution for the equation to be simulated numerically:

$$
u(x, t)=u_{\mathrm{H}}(x, t)-\frac{Q_{\mathrm{F}}}{g(x)} \sum_{k=1}^{p} m_{\mathrm{F}}^{k} \chi_{[x, \xi(x, t)]}\left(x^{k}\right) \exp \left(\mathcal{I}\left(x^{k}, x\right)\right),
$$

where

$$
u_{\mathrm{H}}(x, t)=\frac{g(\xi)}{g(x)} u_{0}(\xi) \exp (\mathcal{I}(\xi, x)), \quad \xi(x, t)= \begin{cases}x \exp \left(-\alpha t / x_{0}\right) & \text { for } \beta=1, \\ {\left[x^{1-\beta}-\frac{\alpha}{x_{0}^{\beta}}(1-\beta) t\right]^{\frac{1}{1-\beta}}} & \text { for } \beta<1 .\end{cases}
$$

Like the solution constructed in Section 2.1, the solution defined by (2.11) is discontinuous, and must be understood as a weak solution, as defined in Appendix A.

We illustrate the solution by considering an initially empty ball mill, $u_{0} \equiv 0$, for which $u_{\mathrm{H}} \equiv 0$, and are particularly interested in the behaviour of the solution for $x \rightarrow 0$. It is straightforward to derive the bounded solution in tensor product form

$$
u(x, t)=-\exp \left(\frac{Q_{\mathrm{S}}}{\alpha}\left(x_{\mathrm{s}}-x\right)\right) \sum_{k=1}^{p} m_{\mathrm{F}}^{k} H\left(-\alpha t-x^{k}\right)
$$

for $\beta=0$ and $x<x_{\mathrm{s}}$. This means that $u(0, t)$ is just a scaled version of the cumulative size distribution of the feed balls, and in particular a piecewise constant function bounded by one.

In the examples plotted here, we chose the parameters $\alpha=-1, x_{0}=1, Q_{\mathrm{F}}=1, x_{\mathrm{s}}=1$, $p=4$, and $x^{k}=1+2 k$ and $m_{\mathrm{F}}\left(x^{k}\right)=0.25$ for $k=1, \ldots, 4$. These values are chosen since they are algebraically simple, but we may easily relate them to real-world parameters as follows. Given that real balls typically are up to four inches in diameter, we may, for example, assume that $x$ is measured in centimeters. If we measure $t$ in tens of days, which is a reasonable time unit in view of the time scale considered in $[13,14]$, then our value of $\alpha$ corresponds to

$$
\alpha=-\frac{1.0 \mathrm{~cm}}{240 \mathrm{~h}}=-0.04167 \mathrm{~mm} / \mathrm{h},
$$



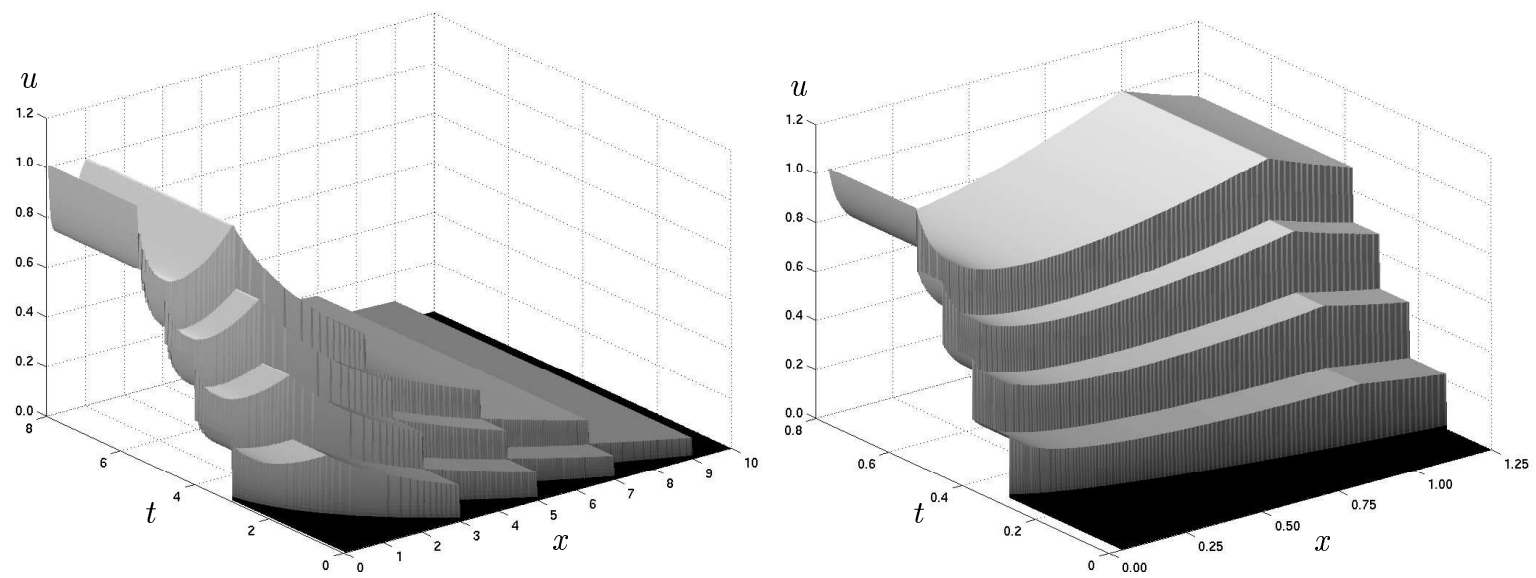

Figure 2. Exact solution of Model 2 with $\beta=0.5$, which becomes unbounded for $x \downarrow 0$ and is plotted for $x \in[0.04,10]$ (left) and $x \in[0.04,1.25]$ (right).

which is a realistic value according to Section 1. Finally, we point out that the number scales of $u, u_{0}, Q_{\mathrm{F}}$ and $Q_{\mathrm{S}}$ must, of course, be the same, but the scale itself can be chosen arbitrarily. For example, if we measure $u$ in thousands of balls per centimeter of size range, then $Q_{\mathrm{F}}=1$ means that we feed 1000 balls per time unit into the ball mill; with the relative number frequencies chosen here and assuming again the sample time scale of ten days, our feed operation consists in continuously feeding 25 balls per day of each of the sizes $3,5,7$ and $9 \mathrm{~cm}$ into the mill. Analogously, choosing, for example, $Q_{\mathrm{S}}=1$ means that at the same time we continuously remove 100 balls per day that have been ground to size $x=x_{\mathrm{s}}=1 \mathrm{~cm}$ or smaller.

Figure 1 shows the solution for this case $(\beta=0)$ with $Q_{\mathrm{S}}=1$.

Next, we consider the range $0<\beta<1$, for which

$$
\lim _{x \downarrow 0} \mathcal{I}\left(x^{k}, x\right)=-\frac{Q_{\mathrm{S}} x_{0}^{\beta} x_{\mathrm{s}}^{1-\beta}}{\alpha(1-\beta)},
$$

which is a well-defined negative constant, and consequently,

$$
\frac{\exp \left(\mathcal{I}\left(x^{k}, x\right)\right)}{g(x)}=\frac{\exp \left(\mathcal{I}\left(x^{k}, x\right)\right)}{x^{\beta}} \longrightarrow-\infty \quad \text { as } x \downarrow 0 .
$$

Moreover,

$$
\xi(0, t)=\left(-\frac{\alpha}{x_{0}^{\beta}}(1-\beta) t\right)^{\frac{1}{1-\beta}} \text { for } 0<\beta<1 .
$$

We obtain $u(0, t)=0$ as long as all summands in (2.10) vanish, that is, as long as $\xi(0, t)<x^{1}$. From (2.14) we deduce that this holds for

$$
t<t^{*}:=-\frac{x_{0}^{\beta}}{\alpha(1-\beta)}\left(x^{1}\right)^{\frac{1}{1-\beta}}
$$



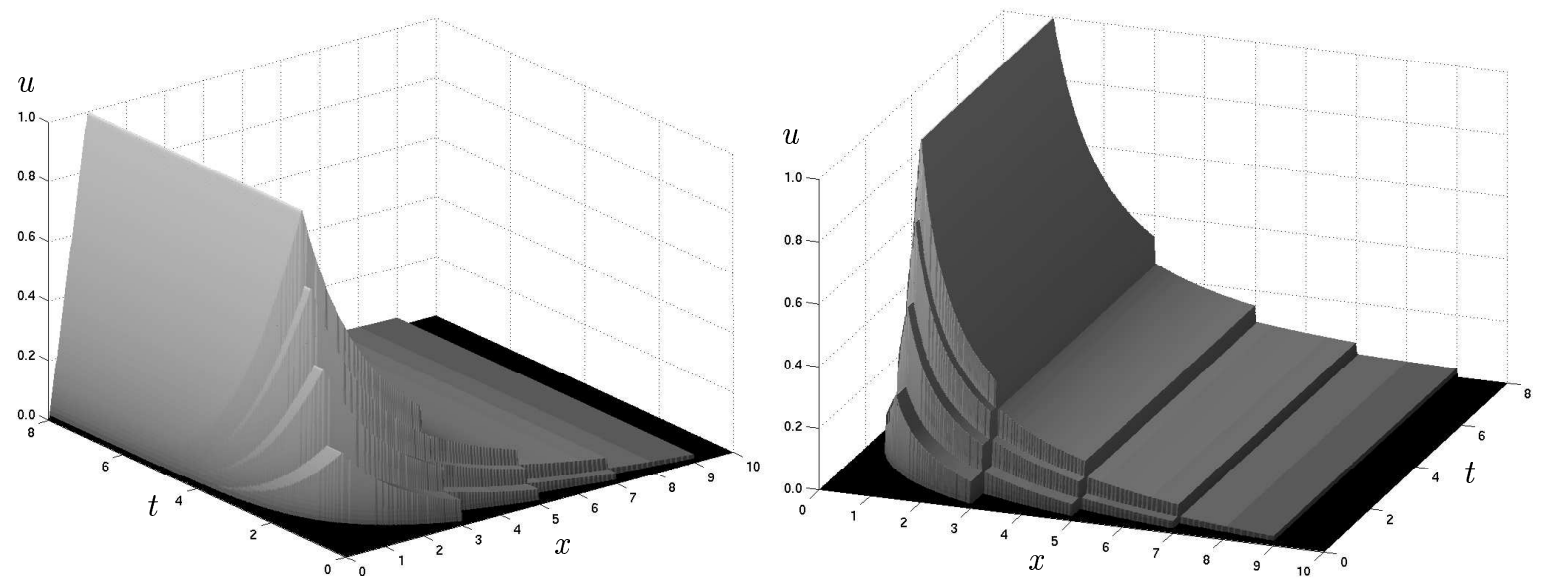

Figure 3. Exact solution of Model 2 with $\beta=1$ and $\alpha>-Q_{\mathrm{S}} x_{0}$.
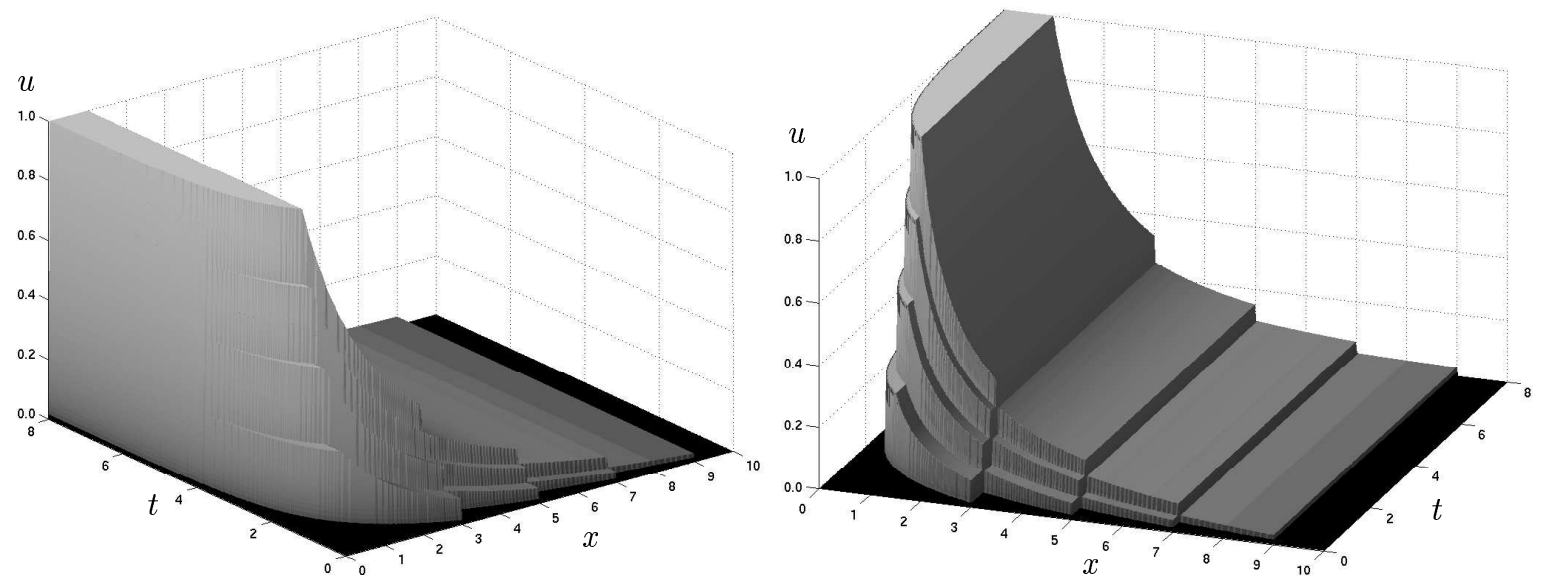

Figure 4. Exact solution of Model 2 with $\beta=1$ and $\alpha=-Q_{\mathrm{S}} x_{0}$.

for $\beta=0.5$ and the remaining parameters as given above, we get $t^{*}=2 \sqrt{3}=3.464$. For $t>t^{*},(2.13)$ implies $u(x, t) \rightarrow \infty$ as $x \downarrow 0$. Figure 2 shows the solution for this case.

For $\beta=1$ and $x<x_{\mathrm{s}}$, we have

$$
\exp \left(\mathcal{I}\left(x^{k}, x\right)\right)=\exp \left(-\frac{Q_{\mathrm{S}} x_{0}}{\alpha} \ln \left(x / x_{\mathrm{S}}\right)\right)=\left(x / x_{\mathrm{S}}\right)^{-\frac{Q_{\mathrm{S}} x_{0}}{\alpha}}
$$

and therefore

$$
u(x, t)=-\frac{Q_{\mathrm{F}} x_{0}}{\alpha} x_{\mathrm{S}}^{\frac{Q_{\mathrm{S}} x_{0}}{\alpha}} x^{-1-\frac{Q_{\mathrm{S}} x_{0}}{\alpha}} \sum_{k=1}^{p} m_{\mathrm{F}}^{k} \chi_{\left[x, x \exp \left(-\alpha t / x_{0}\right)\right]}\left(x^{k}\right) .
$$

For $\alpha>-Q_{\mathrm{S}} x_{0}$, we obtain a continuous function of $x$ that vanishes at $x=0$ and multiplies a finite sum. In this case we have $u(0, t)=0$. 

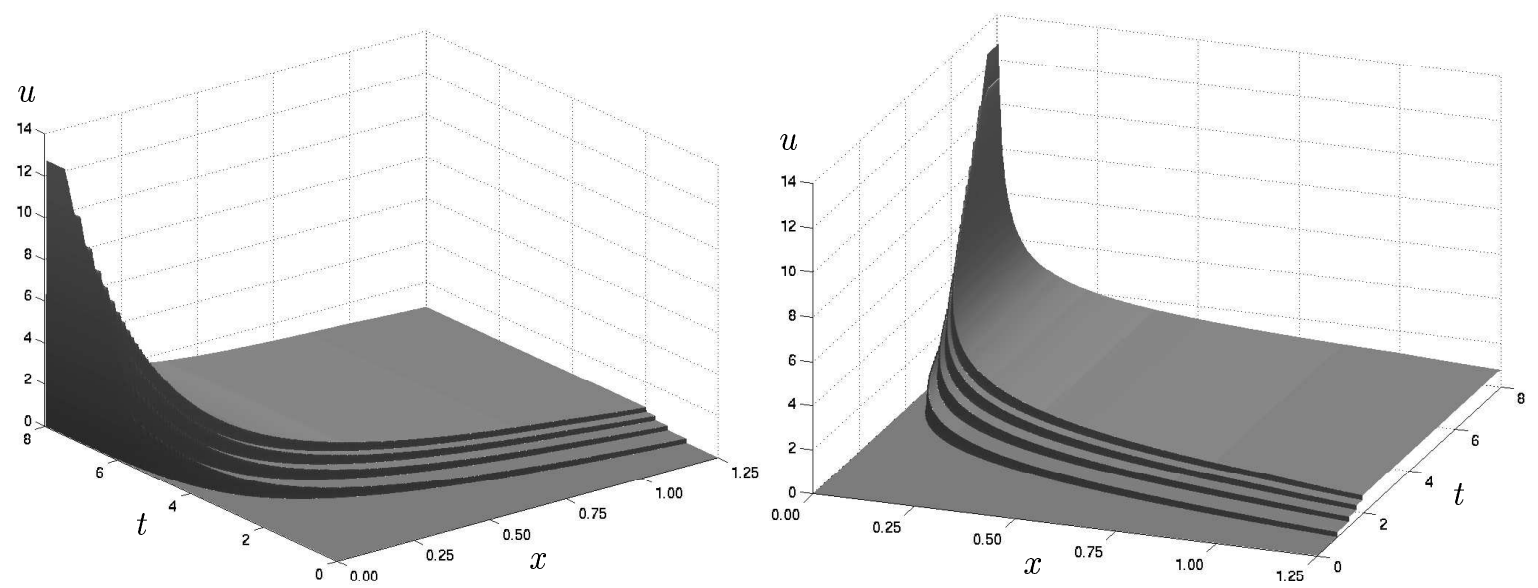

Figure 5. Exact solution of Model 2 with $\beta=1$ and $\alpha<-Q_{\mathrm{S}} x_{0}$.

For $\alpha=-Q_{\mathrm{S}} x_{0}$, we get for $x<x_{\mathrm{S}}$

$$
u(x, t)=\frac{Q_{\mathrm{F}} x_{0}}{Q_{\mathrm{S}} x_{\mathrm{S}}} \sum_{k=1}^{p} m_{\mathrm{F}}^{k} \chi_{\left[x, x \exp \left(Q_{\mathrm{S}} t\right)\right]}\left(x^{k}\right) .
$$

For $x=0$, all the summands in (2.16) are zero, and therefore $u(0, t)=0$. However, it is instructive to note that for any fixed value $x_{*} \in\left(0, x_{\mathrm{s}}\right)$, we have

$$
\lim _{t \rightarrow \infty} u\left(x_{*}, t\right)=\frac{Q_{\mathrm{F}} x_{0}}{Q_{\mathrm{S}} x_{\mathrm{S}}},
$$

since $x^{1}, \ldots, x^{p} \in\left[x_{*}, x_{*} \exp \left(Q_{\mathrm{S}} t\right)\right]$ for $t$ sufficiently large, and thus the value of the sum is $m_{\mathrm{F}}^{1}+\cdots+m_{\mathrm{F}}^{p}=1$.

For $\alpha<-Q_{\mathrm{S}} x_{0}$, the solution at $x=0$ is not defined. The solution becomes unbounded for $x>0$ sufficiently small and $t$ sufficiently large. To see this, fix $x_{*} \in\left(0, x_{\mathrm{s}}\right)$; we then have

$$
u(x, t)>-\frac{Q_{\mathrm{F}} x_{0}}{\alpha} x_{\mathrm{S}}^{\frac{Q_{\mathrm{S}} x_{0}}{\alpha}} x_{*}^{-1-\frac{Q_{\mathrm{S}} x_{0}}{\alpha}} \text { for } x \in\left(0, x_{*}\right) \text { and } t>-\frac{x_{0}}{\alpha} \ln \left(x^{p} / x_{*}\right) .
$$

The solutions for $\beta=0.5$ and the three cases of $\alpha$ are plotted in Figures 3, 4, and 5, respectively, and correspond to $Q_{\mathrm{S}}=2,1$ and 0.5 , and the other parameters as chosen before. We mention that the singular behaviour of the number density function is also well known from population balance models in other applications. See, for example, [8, Section 2.11].

\section{Finite difference schemes}

From here on, our analysis, the development of numerical schemes, and comparisons between exact and numerical solutions are limited to Model 2.

Equation (2.6) can be written as

$$
\frac{\partial u}{\partial t}+\frac{\partial}{\partial x}(g(x) u+\gamma(t) a(x))=b(x, t) u, \quad(x, t) \in \Pi_{T},
$$


where $\Pi_{T}$ is defined in (1.5), $\gamma(t):=-Q_{\mathrm{F}}(t)$, and

$$
a(x):=\sum_{k=1}^{p} m_{\mathrm{F}}^{k} H\left(x-x^{k}\right), \quad b(x, t):=-Q_{\mathrm{S}}(t) \chi_{\left[0, x_{\mathrm{S}}\right]}(x) .
$$

We consider (3.1) together with the initial condition

$$
u(x, 0)=u_{0}(x), \quad x \in(0, X),
$$

and the boundary condition at the right boundary

$$
u(X, t)=0, \quad t \in(0, T] .
$$

No boundary condition is required at $x=0$. Our assumptions on the data are the following:

$$
\begin{aligned}
& g \text { is absolutely continuous on }[0, X], g(x) \leq 0 \text { on }[0, X], g(x)<0 \text { on }(0, X], \\
& \qquad \begin{aligned}
a(x) \geq 0, a \in L^{\infty}((0, X]), a \text { is nondecreasing on }(0, X), \\
a(x) \text { is constant on some interval }\left[X-\delta_{a}, X\right], \text { for some } \delta_{a} \in(0, X], \\
b(x, t) \leq 0, \text { and } b \in L^{\infty}\left(\Pi_{T}\right), \\
\gamma(t) \leq 0, \gamma \in B V([0, T]), \\
u_{0}(x) \geq 0, u_{0} \in B V([0, X]) .
\end{aligned}
\end{aligned}
$$$$
a(x) \geq 0, a \in L^{\infty}((0, X]), a \text { is nondecreasing on }(0, X),
$$

To aid readability, we define $A:=\|a\|_{L^{\infty}}, B:=\|b\|_{L^{\infty}}, \Gamma:=\|\gamma\|_{L^{\infty}}$, and $G:=\|g\|_{L^{\infty}}$.

REMARK 3.1. Concerning condition (3.5), when devising and analysing numerical schemes, we are allowing $g(x)$ to be of slightly more general form than the class of wear rates given by (1.1). Also, we note for future reference that condition (3.5) implies that $g \in B V([0, X])$.

We now turn to the description of our numerical algorithms. In Section 3.1, we construct a first order upwind scheme. In Section 3.2 we modify this first order scheme to achieve improved accuracy. We propose a new design approach, the so-called flux-TVD principle, which we use to derive a reqularizing process that damps out spurious oscillations caused by the second order correction terms. The analysis of our first-order scheme appears in Appendix B, and we defer the analysis of our new second-order scheme to a future paper.

\subsection{First-ORDER SCHEME}

Associated with an integer $J^{\Delta}>0$, define the spatial mesh size $\Delta x=X / J^{\Delta}$, where 'spatial' refers to the internal particle size coordinate. We fix the parameter $\lambda$ and define the temporal mesh size $\Delta t=\lambda \Delta x$, always assuming that the following CFL condition is satisfied:

$$
\lambda G+\Delta t B \leq 1
$$

Let $N^{\Delta}:=\lfloor T / \Delta t\rfloor+1$, so that $T \leq N^{\Delta} \Delta t<T+\Delta t$. The symbol $\Delta$ refers to the discretization defined by $(\Delta x, \Delta t)=(\Delta x, \lambda \Delta x)$. The spatial domain $\mathbb{R}$ is discretized into cells $I_{j}:=\left[x_{j}, x_{j+1}\right)$, where $x_{j}:=j \Delta x$ for $j=0, \ldots J^{\Delta}$. Similarly, the time interval $[0, T]$ is discretized via $t_{n}:=n \Delta t$ for $n=0, \ldots, N$, resulting in the time strips $I^{n}:=\left[t_{n}, t_{n+1}\right)$. We let $\chi_{j}(x)$ and $\chi^{n}(t)$ be the characteristic functions for the intervals $I_{j}$ and $I^{n}$, respectively. 
We let $\chi_{j}^{n}(x, t):=\chi_{j}(x) \chi^{n}(t)$ be the characteristic function for the rectangle $R_{j}^{n}:=I_{j} \times I^{n}$. To simplify the presentation, we use $\Delta_{+}$and $\Delta_{-}$to designate the difference operators in the $x$ direction, e.g., $\Delta_{+} h_{j}^{n}=h_{j+1}^{n}-h_{j}^{n}=\Delta_{-} h_{j+1}^{n}$. Our first-order scheme is then

$$
U_{j}^{n+1}= \begin{cases}U_{j}^{n}-\lambda \Delta_{+}\left(g_{j} U_{j}^{n}+\gamma^{n} a_{j}\right)+\Delta t b_{j}^{n} U_{j}^{n} & \text { for } j \neq J^{\Delta} \\ U_{j}^{n}+\lambda g_{j} U_{j}^{n}+\Delta t b_{j}^{n} U_{j}^{n} & \text { for } j=J^{\Delta} .\end{cases}
$$

For the sake of simplicity, we will always use the first formula, which is possible with the understanding that $U_{J^{\Delta}}^{n}, U_{J^{\Delta}+1}^{n} \equiv 0$ (which enforces the boundary condition (3.4)), and $a_{J \Delta+1} \equiv a_{J \Delta}$. In view of the hypothesis (3.7), this last condition will hold as soon as the mesh size is sufficiently small. Here, we let $g_{j}:=g\left(x_{j}\right), a_{j}:=a\left(x_{j}\right), \gamma^{n}:=\gamma\left(t^{n}\right)$ and $b_{j}^{n}:=b\left(x_{j}, t^{n}\right)$. Note that the scheme (3.12) is one-sided, in particular that it is biased to the right, the direction of incoming information, i.e., the scheme is upwind in particle size and explicit time stepping is used. Recall that we are assuming $g(x) \leq 0$, so that characteristics flow from right to left.

\subsection{SECOND-ORDER SCHEME}

Ignoring for the moment the contribution of the sieve term and the source terms, we see that the scheme proposed in the previous section is a first order upwind scheme for a scalar conservation law. By the end of the 1980's many methods of modifying such first order schemes to achieve higher order accuracy had been proposed [26-28]. The challenge faced by the designers of such schemes is that any naive attempt to improve accuracy will introduce oscillations in regions of rapid transition. For this reason, virtually all of the successful higher order schemes employ some limiting process that reduces the contribution of the higher order correction terms wherever a rapid transition is detected. Usually the limiter is designed in such a way that some regularity property of the actual solution to the conservation law is achieved. This is often sufficient to avoid spurious oscillations, and is not inconsistent with higher order accuracy in regions where the solution is smooth. A number of such schemes have been based on the Total Variation Diminishing (TVD) regularity property of scalar conservation laws. For many scalar conservation laws, the TVD approach achieves a significant increase in accuracy with only moderate additional complexity, and so it is a good candidate for our problem.

Unfortunately, when the flux has discontinuities, as is the case here, it is not reasonable to require the numerical approximations to be TVD, since the solution to the original conservation law will not generally have this property. Instead, we propose the novel approach of requiring that the numerical flux be TVD, because this is a property that the flux for the original conservation law does have. This so-called flux-TVD principal is also motivated by our convergence proof (for the first order scheme) in Appendix B, where the key steps in our compactness proof are bounds on the magnitude and the spatial total variation of the numerical flux. We find that enforcing this flux-TVD property damps out spurious oscillations, but still allows the scheme to have much better accuracy than the first order version. The same idea also applies to nonlinear conservation laws with discontinuous coefficients, which will be the topic of future work.

Let us concentrate on the following equation

$$
\frac{\partial u}{\partial t}+\frac{\partial}{\partial x}(g(x) u+a(x))=b(x) u,
$$


i.e., we ignore all time-dependence of the coefficients for now. The first-order scheme is then

$$
U_{j}^{n+1}=U_{j}^{n}-\lambda \Delta_{+}\left(g_{j} U_{j}^{n}+a_{j}\right)+\Delta t b_{j} U_{j}^{n}=U_{j}^{n}-\lambda \Delta_{+} h_{j}^{n}+\Delta t b_{j} U_{j}^{n} .
$$

For the purpose of a truncation error analysis, we write the scheme as

$$
\frac{U_{j}^{n+1}-U_{j}^{n}}{\Delta t}+\frac{\Delta_{+} h_{j}^{n}}{\Delta x}-b_{j} U_{j}^{n}=0,
$$

and insert a smooth solution, arriving, after a Taylor series expansion, at

$$
\begin{aligned}
\frac{U_{j}^{n+1}-U_{j}^{n}}{\Delta t}+\frac{\Delta_{+} h_{j}^{n}}{\Delta x}-b_{j} U_{j}^{n}= & \frac{1}{\Delta t}\left(\frac{\lambda}{2} \Delta x^{2} \frac{\partial}{\partial x}\left[(1+\lambda g) \frac{\partial}{\partial x}(g u+a)\right]-\frac{\lambda}{2} \Delta t \Delta x \frac{\partial}{\partial x}(g b u)\right. \\
& \left.-\frac{1}{2} \Delta t \cdot b\left[\lambda \Delta x \frac{\partial}{\partial x}(g u+a)-\Delta t b u\right]\right)+\mathcal{O}\left(\Delta x^{2}\right) .
\end{aligned}
$$

In deriving this formula for the truncation error, we have used (3.13) to replace $u_{t}$, and, to replace the term $\partial^{2} u / \partial t^{2}$, the relationship

$$
\frac{\partial^{2} u}{\partial t^{2}}=\frac{\partial}{\partial x}\left(g\left[\frac{\partial}{\partial x}(g u+a)-b u\right]\right)-b\left[\frac{\partial}{\partial x}(g u+a)-b u\right] .
$$

For the moment, let us ignore the sieve portion of the model, leaving us with the conservation law

$$
\frac{\partial u}{\partial t}+\frac{\partial}{\partial x}(g(x) u+a(x))=0 .
$$

By closely examining the analysis in the previous section, one can see that the first order scheme for this PDE,

$$
U_{j}^{n+1}=U_{j}^{n}-\lambda \Delta_{+} h_{j}^{n},
$$

has the property that the numerical flux $h_{j}^{n}$ is TVD:

$$
\sum_{j=1}^{J^{\Delta}}\left|\Delta_{-} h_{j}^{n+1}\right| \leq \sum_{j=1}^{J^{\Delta}}\left|\Delta_{-} h_{j}^{n}\right|,
$$

the property that we referred to above as flux-TVD. The scheme (3.16) approximates the conservation law (3.15) to first order, and the truncation error equals the first term on the right-hand side of (3.14), i.e.

$$
\frac{1}{\Delta t} \frac{\lambda}{2} \Delta x^{2} \frac{\partial}{\partial x}\left((1+\lambda g) \frac{\partial}{\partial x}(g u+a)\right) .
$$

To construct a second-order scheme for (3.15), we will add flux corrections to (3.16), generating a Lax-Wendroff type scheme of the type described in [29], and then apply bounding functions, referred to in the conservation law literature as limiters, to enforce the flux-TVD property. The second-order version of (3.16) will take the form

$$
U_{j}^{n+1}=U_{j}^{n}-\lambda \Delta_{+}\left(h_{j}^{n}+\hat{e}_{j-1 / 2}^{n}\right),
$$


where $\hat{e}_{j-1 / 2}^{n}$ is a regularized version of the second-order correction $e_{j-1 / 2}^{n}$. Here the ^ notation indicates that some limiter has been applied. A straightforward calculation reveals that for our simplified scheme (3.19) with the correction terms present, the first-order flux evolves according to

$$
h_{j}^{n+1}=h_{j}^{n}-\lambda g_{j}\left(1+\frac{\Delta_{+} \hat{e}_{j-1 / 2}^{n}}{\Delta_{+} h_{j}^{n}}\right) \Delta_{+} h_{j}^{n} .
$$

From this equation it is clear that the flux-TVD property (3.17) as well as a maximum principle for the numerical flux hold if

$$
0 \leq-\lambda g_{j}\left(1+\frac{\Delta_{+} \hat{e}_{j-1 / 2}^{n}}{\Delta_{+} h_{j}^{n}}\right) \leq 1 .
$$

Guided by the truncation error term (3.18), we let

$$
e_{j+1 / 2}^{n}=-\frac{1}{2}\left(1+\lambda g_{j}\right) \Delta_{+} h_{j}^{n}
$$

If we impose the CFL condition (recall that $g_{j} \leq 0$, and that we are ignoring the sieve term for now) $-\lambda g_{j} \leq 1$, then the condition (3.20) will be satisfied if we apply the limiter

$$
\hat{e}_{j-1 / 2}^{n}=\operatorname{minmod}\left\{e_{j+1 / 2}^{n}, e_{j-1 / 2}^{n}\right\}, \quad j=1, \ldots, J^{\Delta}-1 .
$$

It is clear that wherever the solution is smooth, application of the limiter in (3.21) will not destroy the formal second-order accuracy. One detail that needs some attention here is that (3.21) is not well-defined for $j=0$. In the numerical examples we have set $\hat{e}_{-1 / 2}=\hat{e}_{1 / 2}$.

For the sieve portion of the scheme the formula for the truncation error (3.14) suggests that the correction term should be

$$
-\frac{\lambda}{2} \Delta t \Delta_{+}\left(g_{j} b_{j} U_{j}^{n}\right)-\frac{1}{2} \Delta t b_{j}\left(\lambda \Delta_{+} h_{j}^{n}-\Delta t b_{j} U_{j}^{n}\right) .
$$

With the sieve corrections included, the second order scheme is then

$$
\begin{aligned}
U_{j}^{n+1}= & U_{j}^{n}-\lambda \Delta_{+}\left(h_{j}^{n}+\hat{e}_{j-1 / 2}^{n}\right) \\
& +\Delta t b_{j} U_{j}^{n}-\frac{\lambda}{2} \Delta t \Delta_{+}\left(g_{j} b_{j} U_{j}^{n}\right)-\frac{1}{2} \Delta t b_{j}\left(\lambda \Delta_{+} h_{j}^{n}-\Delta t b_{j} U_{j}^{n}\right) .
\end{aligned}
$$

Our numerical experiments (see Section 4) indicate that this second order version is well-behaved, i.e., it suffices to apply limiters to the flux corrections, and no additional regularization processing is required for the second order corrections applied to the sieve term. Although we have not done so here, the flux-TVD approach for constructing second order schemes described above can be put on a rigorous theoretical footing, even for nonlinear conservation laws with discontinuous coefficients. We defer that analysis to a later paper.

\section{Numerical simulations}

To test the two numerical schemes, we select three of the five cases for which an exact solution is presented in Section 2.3, and utilize the same parameters. Specifically, we restrict ourselves 

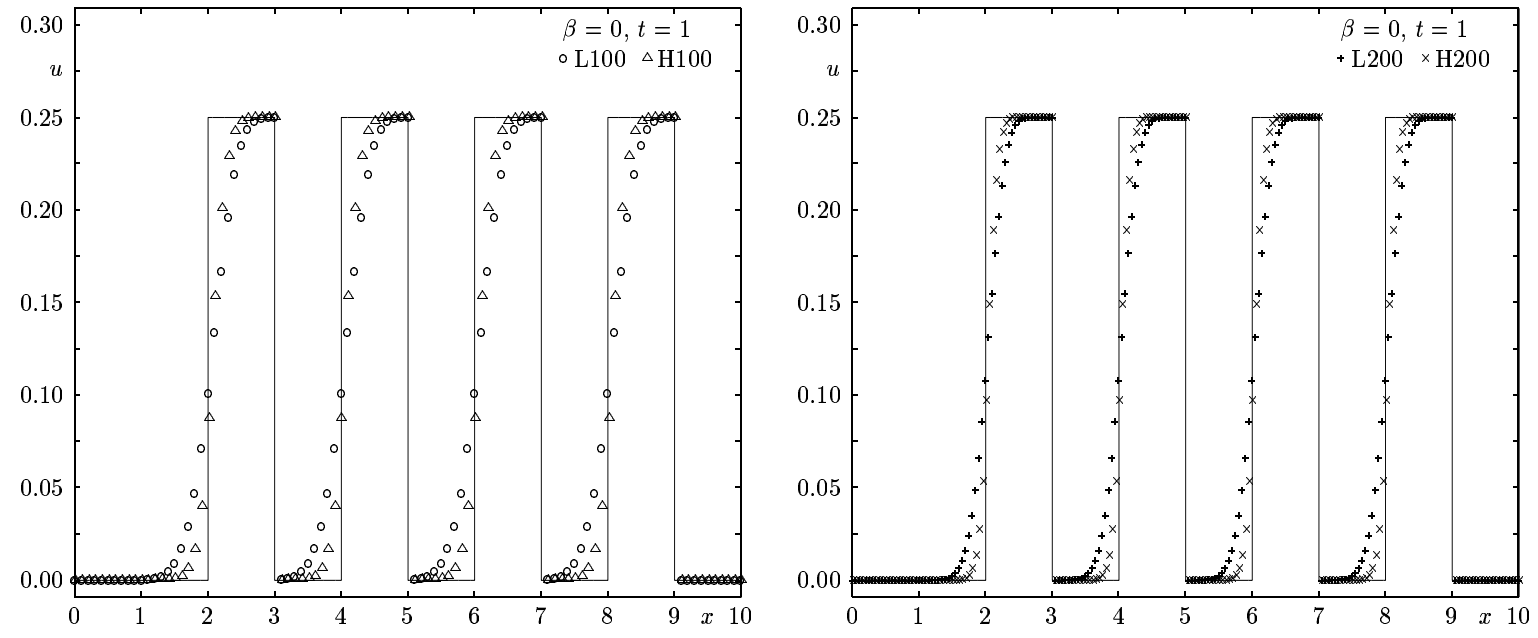

Figure 6. Numerical simulation of a ball mill with $\beta=0$ at $t=1$. Here and in Figures $7-14$, numerical solutions are depicted by symbols and the exact solution by a solid line, and ' $\mathrm{L}$ ' and ' $\mathrm{H}$ ' refer to the low and high order schemes used with the indicated value of $J^{\Delta}$, respectively.
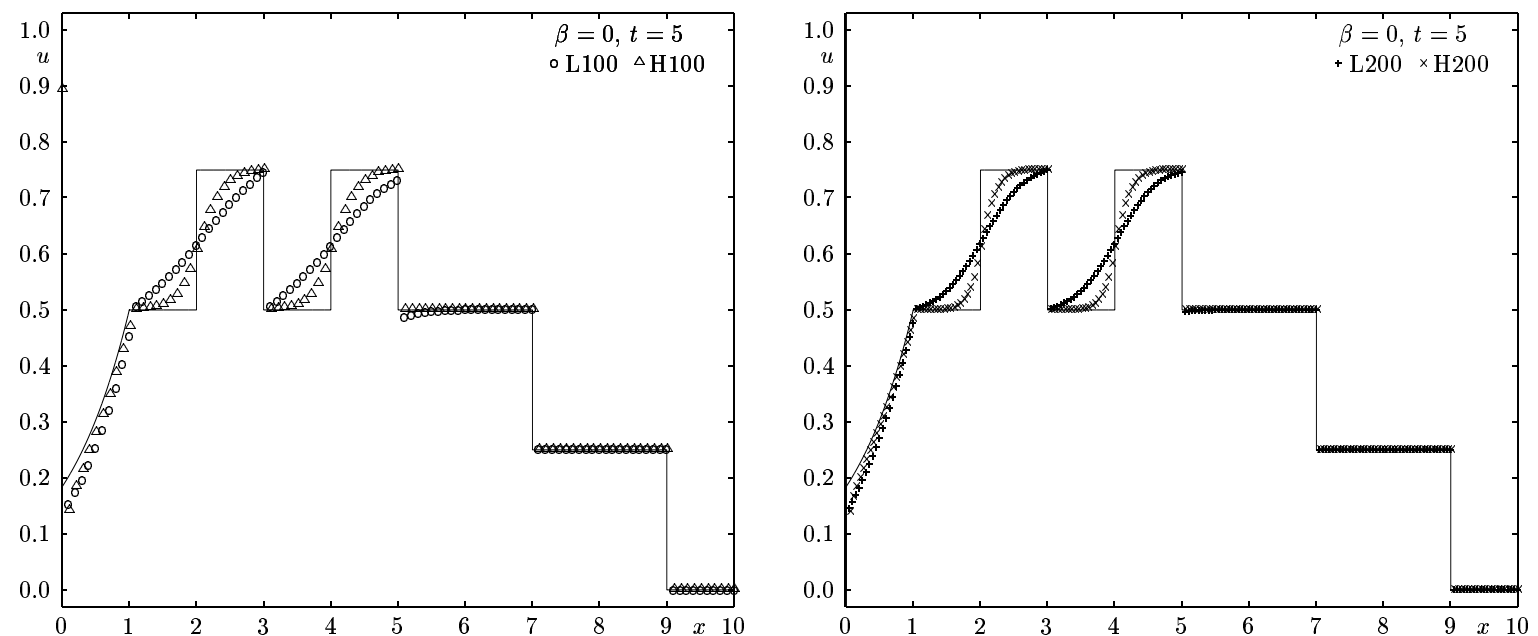

Figure 7. Numerical simulation of a ball mill with $\beta=0$ at $t=5$.

to $\alpha=-Q_{\mathrm{S}} x_{0}$ and consider the solutions plotted in Figures 1,2 and 4, corresponding to $\beta=0, \beta=0.5$, and $\beta=1$, respectively. Consequently, the test cases differ in the value of $\beta$ only. Figures $6-14$ show the numerical results for these three cases, corresponding to solution profiles at times $t=1, t=5$ and $t=10$ in each case. The numerical solutions have been produced by using $\lambda=0.1$ and the discretizations $\Delta x=X / J^{\Delta}$ with $X=10$ and $J^{\Delta}=100$ for the left and $J^{\Delta}=200$ for the right diagram in each of these figures. The runs with the lowaccuracy first-order and the high-accuracy second-order scheme are denoted by L100, L200 and H100, H200, respectively. To record an error history and to measure convergence rates, we have performed additional simulations with one coarser and several finer discretizations and measured the $L^{1}$ errors and convergence rates listed in Tables I-III. 

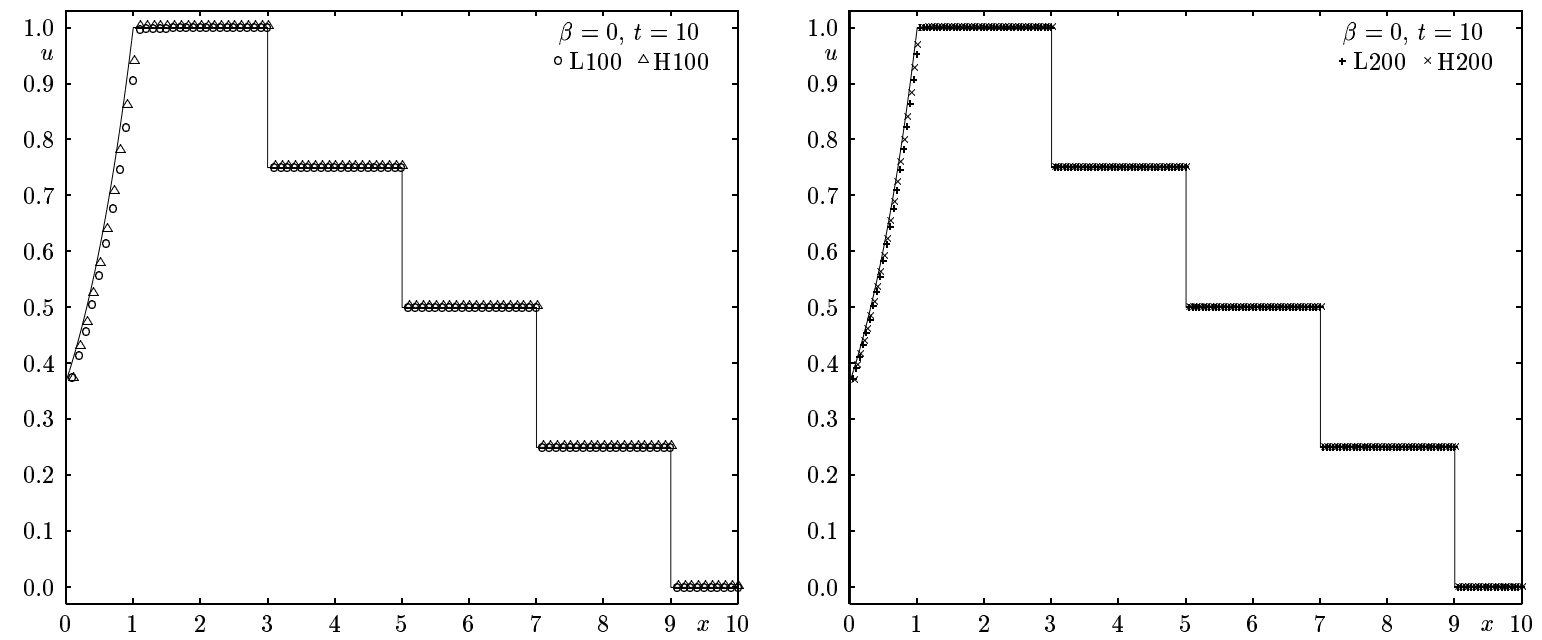

Figure 8. Numerical simulation of a ball mill with $\beta=0$ at $t=10$.
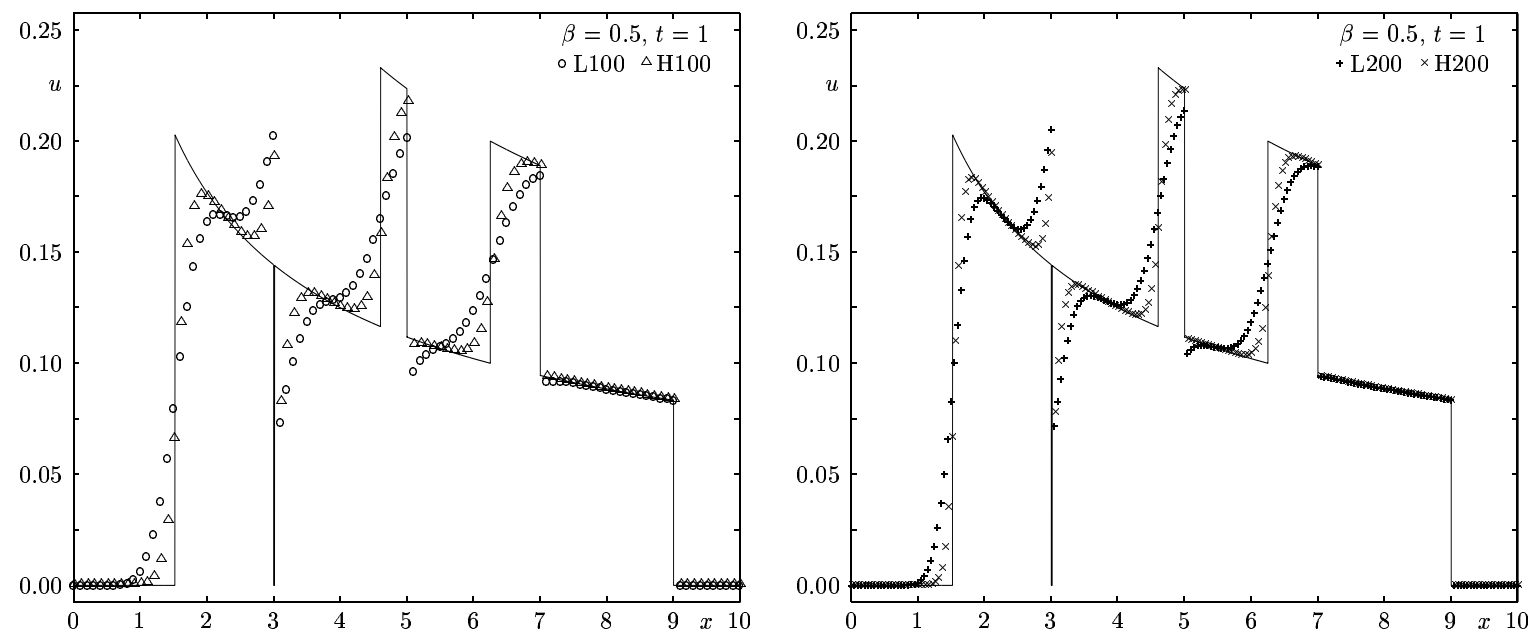

Figure 9. Numerical simulation of a ball mill with $\beta=0.5$ at $t=1$.

Figure 6 shows that stationary discontinuities due to the jumps in $a(x)$ are sharply resolved, while the traveling discontinuities located around $x=2,4,6$ and 8 are smeared out, which is a well-known property of standard monotone schemes for conservation laws. As expected, discontinuities are more sharply resolved by the second-order scheme. This also becomes visible in Figure 7. Moreover, the leftmost numerical solution points (corresponding to $x=0$ ) attain high values that increase with $J^{\Delta}$; for example, at $t=1$ we have $u_{0}^{n}>1$ for the H200 scheme. These singular solution values are not plotted in this and subsequent figures. Figure 8 shows that the steady-state solution is approximated well by both families of schemes. However, a thin numerical boundary layer produced by high solution values at $x=0$ persists.

A new feature that can bee seen in Figure 9 is a very narrow gap near $x=3$, which closes a short time later on. The numerical solution exhibits a jump of nearly the same strength (near $x=3$ ), but which is shifted upwards. Furthermore, we see for example that information 

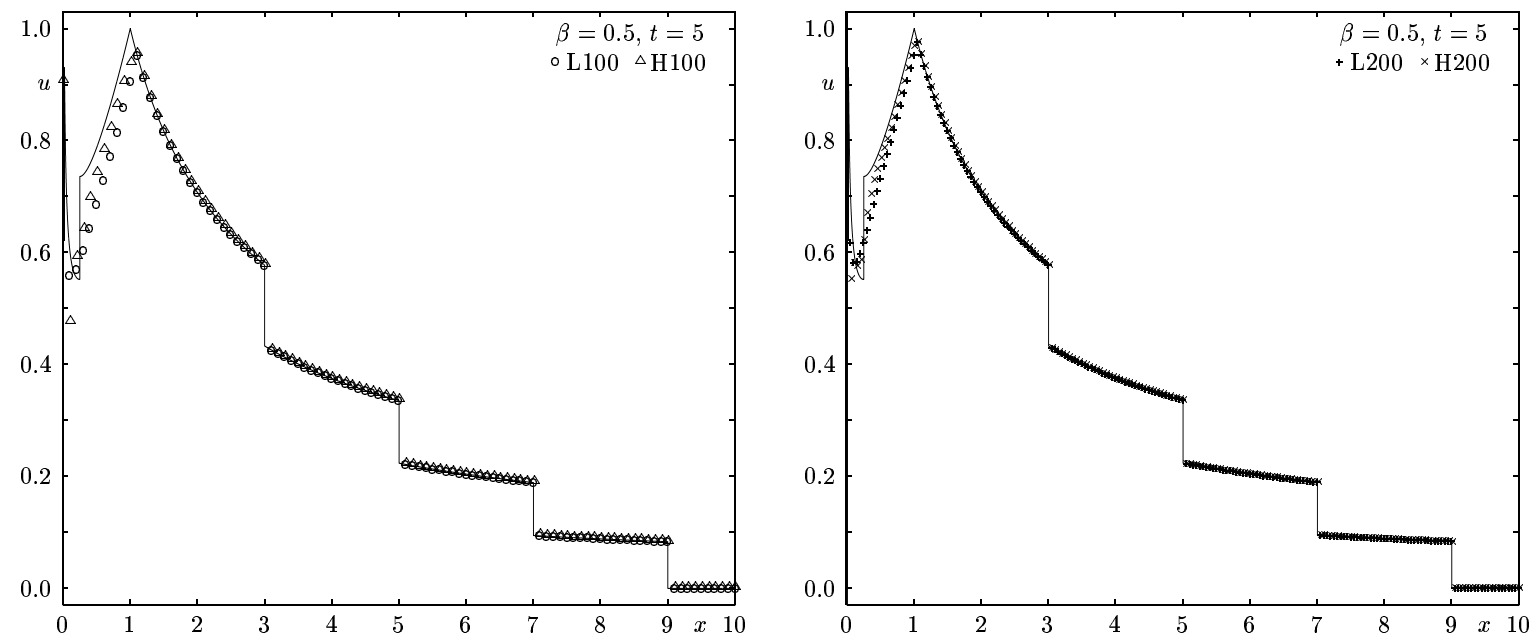

Figure 10. Numerical simulation of a ball mill with $\beta=0.5$ at $t=5$.
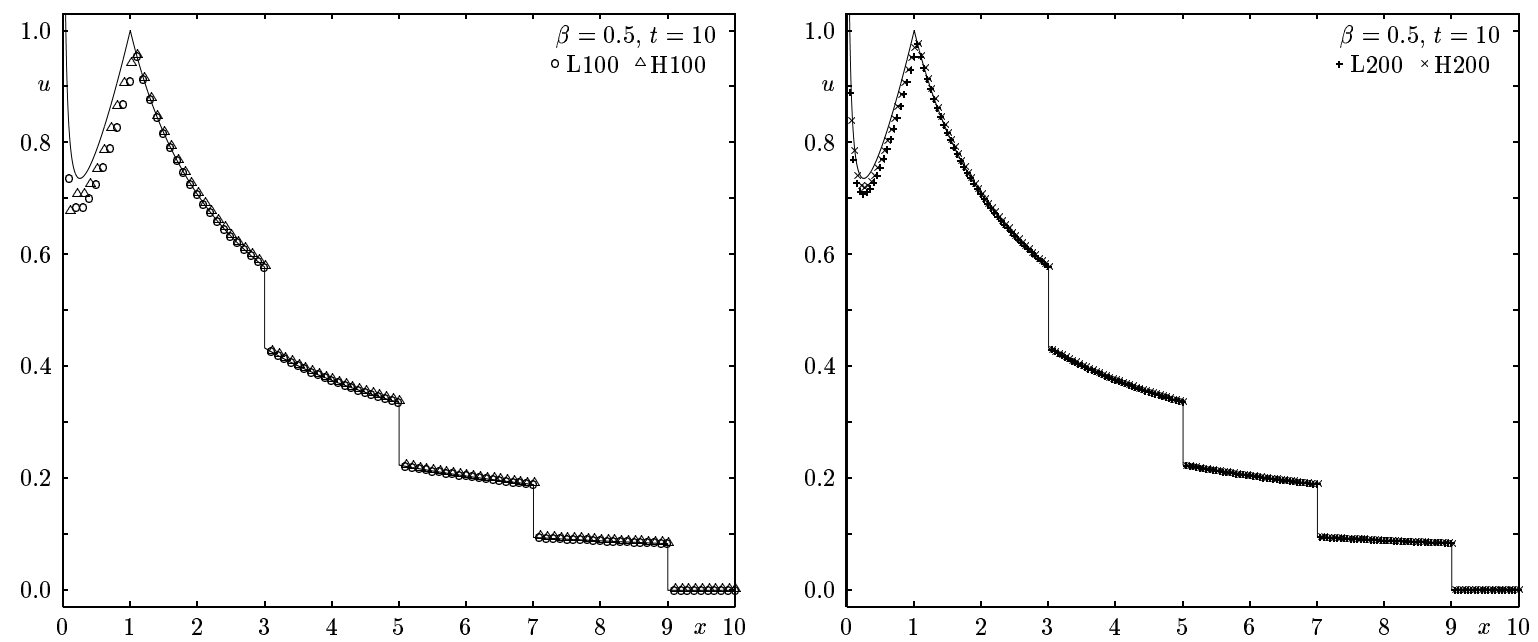

Figure 11. Numerical simulation of a ball mill with $\beta=0.5$ at $t=10$.

traveling downstream from $x^{1}=9$ has already surpassed the next smaller discontinuity of $a(x)$ at $x^{2}=7$, so that the discontinuity of the numerical solution around $x^{3}=7$ (and, similarly, around $x=x^{2}=5$ ) is sharp, but the adjacent numerical values are too small. This spurious effect decreases with increasing accuracy, however. Figure 10 displays a situation in which the exact solution for has attained steady state for $x>x_{\mathrm{s}}=1$ (see also Figure 4). Figure 9 illustrates that the second-order scheme is significantly more accurate on the interval $(0,1]$ than the first-order version. This tendency is also reflected by the lower $L^{1}$ errors for the second-order scheme listed in the middle column of Table II. The superiority of the second-order scheme is also visible in the very similar Figure 11.

Unlike what we have seen in the previous two cases, the choice $\beta=1$ does not lead to numerical solutions that become unbounded near $x=0$. Figure 12 displays an exact solution profile with a number of sawtooth-shaped extrema. As expected, these 'peaks' are poorly 

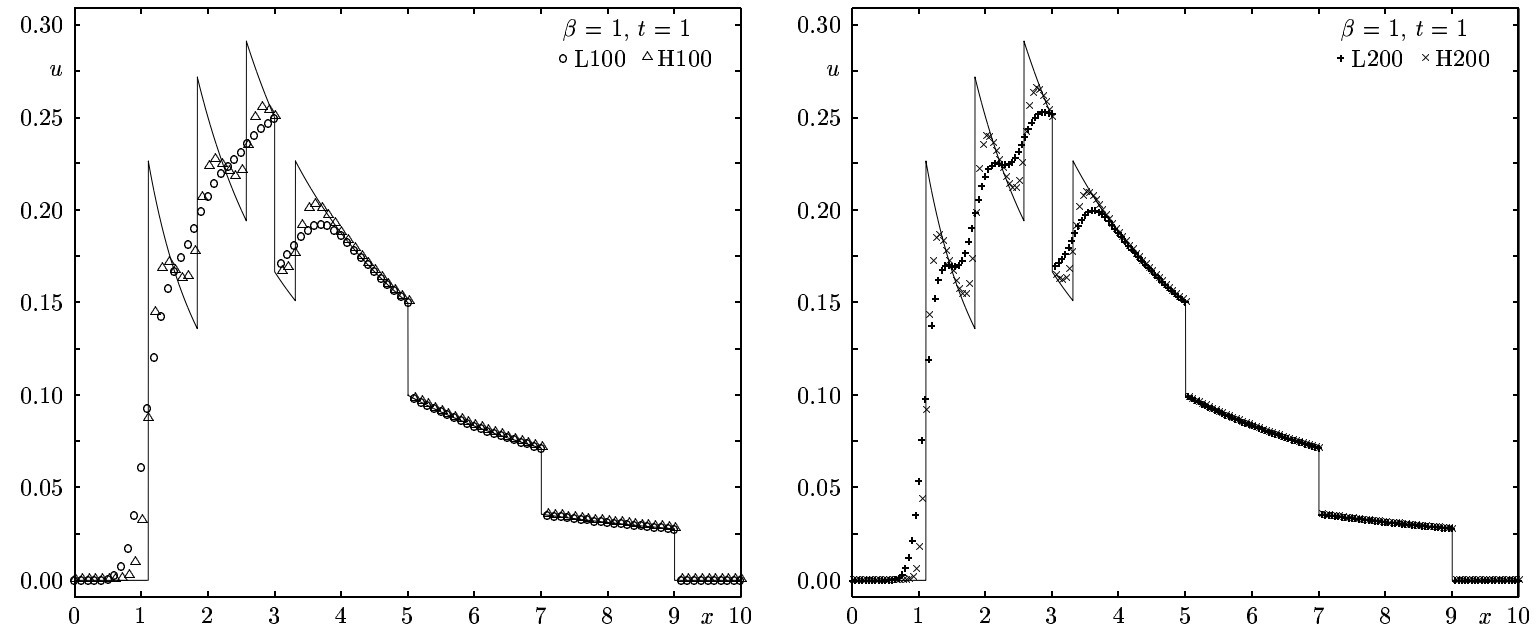

Figure 12. Numerical simulation of a ball mill with $\beta=1$ at $t=1$.
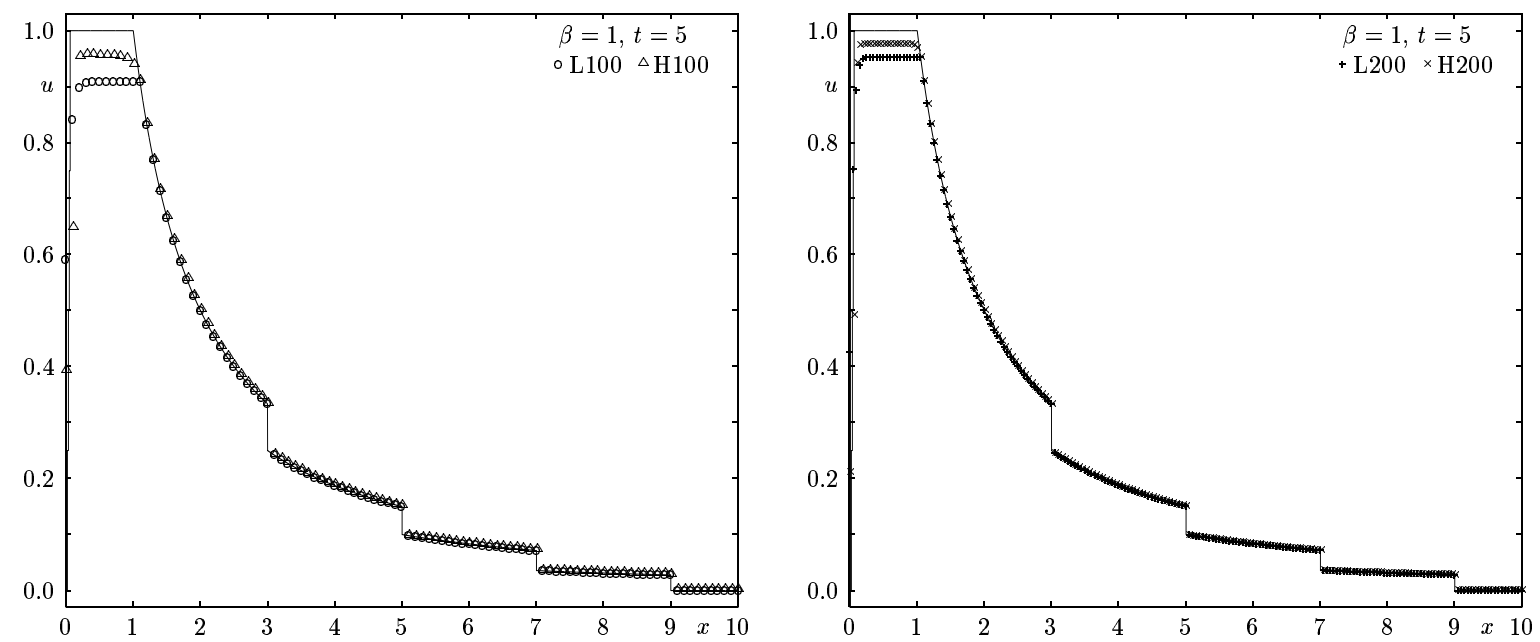

Figure 13. Numerical simulation of a ball mill with $\beta=1$ at $t=5$.

approximated by the first-order scheme. The discontinuity at $x^{4}=3$ is sharply resolved, however. Figures 13 and 14 differ only in the solution near $x=0$. The superiority of the second-order scheme becomes apparent in the error history recorded in Table III, and is also visible in these two figures in that the exact maximal solution value one is better approximated by the second-order scheme than by the first-order scheme with the same value of $J^{\Delta}$.

\section{Appendix A. Weak solutions and uniqueness}

With $g(x)$ allowed to vanish at the origin, solutions of Model 2 are not necessarily bounded near $x=0$. Physically, this corresponds to an accumulation of balls that have been ground down to a size close to zero. To accommodate this lack of a bound, it is natural to work with 

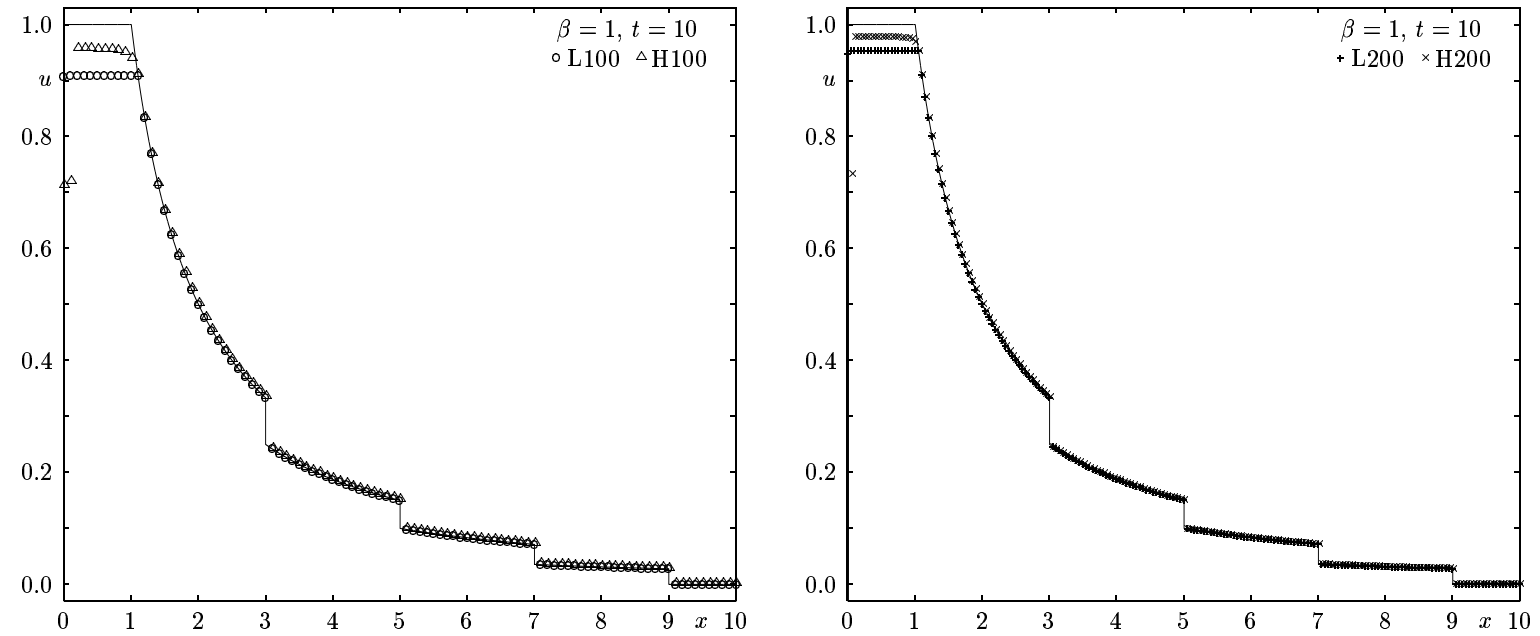

Figure 14. Numerical simulation of a ball mill with $\beta=1$ at $t=10$.

Table I. $L^{1}$ errors for $\beta=0$.

\begin{tabular}{|c|c|c|c|c|c|c|}
\hline \multirow{3}{*}{$J=\frac{\mid 7}{\Delta x}$} & \multicolumn{6}{c|}{ Scheme 1} \\
\cline { 2 - 7 } & \multicolumn{2}{|c|}{$t=1$} & \multicolumn{2}{c|}{$t=5$} & \multicolumn{2}{c|}{$t=10$} \\
\cline { 2 - 7 } & $L^{1}$ error & $\begin{array}{c}\text { conv. } \\
\text { rate }\end{array}$ & $L^{1}$ error & $\begin{array}{c}\text { conv. } \\
\text { rate }\end{array}$ & $L^{1}$ error & $\begin{array}{c}\text { conv. } \\
\text { rate }\end{array}$ \\
\hline 50 & 0.58122 & & 0.57302 & & 0.37836 & \\
100 & 0.35585 & 0.708 & 0.40295 & 0.508 & 0.18758 & 1.012 \\
200 & 0.22515 & 0.660 & 0.27644 & 0.544 & 0.09223 & 1.024 \\
400 & 0.14677 & 0.617 & 0.18353 & 0.591 & 0.04567 & 1.014 \\
800 & 0.09787 & 0.585 & 0.12209 & 0.588 & 0.02305 & 0.986 \\
1600 & 0.06636 & 0.561 & 0.08256 & 0.564 & 0.01212 & 0.927 \\
2000 & 0.05873 & 0.548 & 0.07305 & 0.549 & 0.01004 & 0.841 \\
\hline & \multicolumn{7}{|c|}{ Scheme 2} & & \\
50 & 0.48088 & & 0.48776 & & 0.32963 & \\
100 & 0.26259 & 0.873 & 0.27157 & 0.845 & 0.16402 & 1.007 \\
200 & 0.14687 & 0.838 & 0.15238 & 0.834 & 0.08188 & 1.002 \\
400 & 0.08400 & 0.806 & 0.08691 & 0.810 & 0.04101 & 0.997 \\
800 & 0.04898 & 0.778 & 0.05039 & 0.786 & 0.02072 & 0.984 \\
1600 & 0.02904 & 0.754 & 0.02970 & 0.763 & 0.01082 & 0.937 \\
2000 & 0.02462 & 0.741 & 0.02515 & 0.745 & 0.00893 & 0.860 \\
\hline
\end{tabular}

the weighted $L^{1}$ norm

$$
\|w\|_{L_{g}^{1}([0, X])}:=\int_{0}^{X}|w(x) \| g(x)| d x .
$$

We use the notation $L_{g}^{1}([0, X])$ for the Banach space of measurable functions on $[0, X]$ for which this norm is finite. Solutions of Model 2 are also not generally continuous and thus need to be defined as weak solutions. For this purpose we introduce the space of test functions

$$
\mathcal{D}:=\left\{\psi \in C\left(\bar{\Pi}_{T}\right) \mid \psi(0, t)=0, \psi(x, T)=0, \frac{\partial \psi}{\partial x} \in L^{1}\left(\Pi_{T}\right), \frac{\partial \psi}{\partial t} \in L^{\infty}\left(\Pi_{T}\right)\right\} .
$$


Table II. $L^{1}$ errors for $\beta=0.5$.

\begin{tabular}{|c|c|c|c|c|c|c|}
\hline \multirow{3}{*}{$J=\frac{\mid 7}{\Delta x}$} & \multicolumn{6}{|c|}{ Scheme 1 } \\
\cline { 2 - 7 } & \multicolumn{2}{|c|}{$t=1$} & \multicolumn{2}{c|}{$t=5$} & \multicolumn{2}{c|}{$t=10$} \\
\cline { 2 - 7 } & & conv. & & conv. \\
rate & $L^{1}$ error & rate & $L^{1}$ error & $\begin{array}{c}\text { conv. } \\
\text { rate }\end{array}$ \\
\hline 50 & 0.23582 & & 0.32149 & & 0.27982 & \\
100 & 0.17632 & 0.420 & 0.16958 & 0.923 & 0.14501 & 0.948 \\
200 & 0.12419 & 0.506 & 0.09368 & 0.856 & 0.07457 & 0.960 \\
400 & 0.08611 & 0.528 & 0.05545 & 0.757 & 0.03948 & 0.918 \\
800 & 0.05965 & 0.530 & 0.03570 & 0.635 & 0.02154 & 0.874 \\
1600 & 0.04148 & 0.521 & 0.02373 & 0.589 & 0.01212 & 0.830 \\
2000 & 0.03667 & 0.552 & 0.02123 & 0.500 & 0.01013 & 0.803 \\
\hline & \multicolumn{7}{|c|}{ Scheme 2} \\
50 & 0.19033 & & 0.27433 & & 0.25013 & \\
100 & 0.11657 & 0.707 & 0.13859 & 0.985 & 0.12522 & 0.998 \\
200 & 0.07083 & 0.719 & 0.07461 & 0.894 & 0.06056 & 1.048 \\
400 & 0.04254 & 0.735 & 0.03945 & 0.919 & 0.03206 & 0.918 \\
800 & 0.02641 & 0.688 & 0.02088 & 0.918 & 0.01827 & 0.811 \\
1600 & 0.01601 & 0.722 & 0.01275 & 0.712 & 0.01072 & 0.769 \\
2000 & 0.01340 & 0.796 & 0.01107 & 0.635 & 0.00909 & 0.743 \\
\hline
\end{tabular}

Table III. $L^{1}$ errors for $\beta=1$.

\begin{tabular}{|c|c|c|c|c|c|c|}
\hline \multirow{3}{*}{$J=\frac{\mid 7}{\Delta x}$} & \multicolumn{6}{|c|}{ Scheme 1} \\
\cline { 2 - 7 } & \multicolumn{2}{|c|}{$t=1$} & \multicolumn{2}{c|}{$t=5$} & \multicolumn{2}{c|}{$t=10$} \\
\cline { 2 - 7 } & $L^{1}$ error & $\begin{array}{c}\text { conv. } \\
\text { rate }\end{array}$ & $L^{1}$ error & $\begin{array}{c}\text { conv. } \\
\text { rate }\end{array}$ & $L^{1}$ error & $\begin{array}{c}\text { conv. } \\
\text { rate }\end{array}$ \\
\hline 50 & 0.14028 & & 0.25857 & & 0.25416 & \\
100 & 0.11202 & 0.324 & 0.15020 & 0.784 & 0.14217 & 0.838 \\
200 & 0.08488 & 0.400 & 0.08648 & 0.796 & 0.07533 & 0.916 \\
400 & 0.06113 & 0.474 & 0.04720 & 0.873 & 0.03880 & 0.957 \\
800 & 0.04359 & 0.488 & 0.02655 & 0.830 & 0.01972 & 0.977 \\
1600 & 0.03011 & 0.533 & 0.01436 & 0.887 & 0.00998 & 0.983 \\
2000 & 0.02721 & 0.453 & 0.01250 & 0.620 & 0.00803 & 0.977 \\
\hline & \multicolumn{7}{|c|}{ Scheme 2} \\
50 & 0.13033 & & 0.22822 & & 0.22465 & \\
100 & 0.08417 & 0.631 & 0.12984 & 0.814 & 0.12260 & 0.874 \\
200 & 0.05066 & 0.732 & 0.07550 & 0.782 & 0.06424 & 0.932 \\
400 & 0.03105 & 0.707 & 0.03961 & 0.931 & 0.03292 & 0.965 \\
800 & 0.01928 & 0.688 & 0.01985 & 0.996 & 0.01667 & 0.982 \\
1600 & 0.01133 & 0.767 & 0.01025 & 0.953 & 0.00842 & 0.985 \\
2000 & 0.00999 & 0.564 & 0.00874 & 0.713 & 0.00677 & 0.973 \\
\hline
\end{tabular}

DEFINITION 5.1 (Weak solution). A function $u \in C\left([0, T] ; L_{g}^{1}([0, X])\right.$ is called a weak solution of the initial-boundary value problem defining Model 2, (3.1), (3.3) and (3.4), if

$$
g(x) u+\gamma(t) a(x) \in L^{\infty}\left(\Pi_{T}\right)
$$


and the following weak formulation of the problem is satisfied, for all test functions $\phi \in \mathcal{D}$ :

$$
\begin{aligned}
& \int_{0}^{T} \int_{0}^{X}\left\{u \frac{\partial \phi}{\partial t}+(g(x) u+\gamma(t) a(x)) \frac{\partial \phi}{\partial x}+b(x, t) u \phi\right\} d x d t \\
& -\int_{0}^{T} \gamma(t) a(X) \phi(X, t) d t+\int_{0}^{X} u_{0}(x) \phi(x, 0) d x=0 .
\end{aligned}
$$

The closed-form solutions to Model 2 that we derived in Section 2.3 are weak solutions in the sense of Definition 5.1. In Appendix B, we will show that the limits of a certain difference scheme are also weak solutions. We would like some assurance that the closed-form solutions and the limit of numerical solutions are identical, which leads us to consider the question of uniqueness. For the sake of simplicity, we restrict the analysis to the case where the sieve term $b$ is independent of time, $b=b(x)$.

THEOREM 5.1 (Uniqueness). Assume that the sieve term is independent of time, i.e., $b=$ $b(x)$, and that $1 / g \in L^{1}([0, X])$. If $u$ and $v$ are two weak solutions having the same initial data $u_{0}(x)$, then $u(x, t)=v(x, t)$ a.e. in $\Pi_{T}$.

REMARK 5.1. With the assumption $1 / g \in L^{1}([0, X])$, we are including all of the wear models of the type (1.1) with $0 \leq \beta<1$.

Proof. Let $u$ and $v$ be a pair of weak solutions having the same initial data. Let $w:=v-u$ denote their difference, and observe that due to the linearity of the problem, $w$ satisfies

$$
\int_{0}^{T} \int_{0}^{X} w\left(\frac{\partial \psi}{\partial t}+g(x) \frac{\partial \psi}{\partial x}+b(x) \psi\right) d x d t=0
$$

for all test functions $\psi \in \mathcal{D}$. If we can show that the backward initial-boundary value problem

$$
\frac{\partial \psi}{\partial t}+g(x) \frac{\partial \psi}{\partial x}+b(x) \psi=\phi(x, t) \quad \text { for a.e. }(x, t) \in \Pi_{T}, \quad \psi(0, t)=0, \quad \psi(x, T)=0
$$

has a solution $\psi \in \mathcal{D}$ for all $C^{\infty}$ functions $\phi$ having support in $\Pi_{T}$, then by substituting $\psi$ into (5.3), we will have

$$
\int_{0}^{T} \int_{0}^{X} w \phi d x d t=0
$$

implying that $w=0$ a.e. in $\Pi_{T}$.

In fact, an explicit solution to (5.4) is given by

$$
\psi(x, t)=\int_{0}^{T-t} z(x, t, \tau) d \tau
$$

where

$$
z(x, t, \tau):=-\phi(\hat{y}, T-\tau) \exp \left(\int_{x}^{\hat{y}} \frac{b(s)}{g(s)} d s\right), \quad \hat{y}:=y(x, T-t-\tau) .
$$

Here, $y \in C\left(\bar{\Pi}_{T}\right) \cap C^{1}\left(\Pi_{T}\right)$ is a classical solution to the initial-boundary value problem

$$
\frac{\partial y}{\partial t}-g(x) \frac{\partial y}{\partial x}=0, \quad y(x, 0)=x, \quad y(0, t)=0 .
$$


If we let

$$
P(x):=-\int_{0}^{x} \frac{d w}{g(w)}, \quad \Pi(t):=P^{-1}(t),
$$

then a specific formula for $y$ is given by

$$
y(x, t)= \begin{cases}\Pi(P(x)-t) & \text { for } t<P(x), \\ 0 & \text { for } t \geq P(x) .\end{cases}
$$

We will also need the partial derivatives of $y(x, t)$; for convenience, we denote partial derivatives in the remainder of this proof by $y_{t}, y_{x}$, and so on. We get

$$
y_{t}(x, t)= \begin{cases}g(\Pi(P(x)-t)) & \text { for } t<P(x) \\ 0 & \text { for } t \geq P(x)\end{cases}
$$

and

$$
y_{x}(x, t)= \begin{cases}g(\Pi(P(x)-t)) / g(x) & \text { for } t<P(x), \\ 0 & \text { for } t \geq P(x)\end{cases}
$$

where we have used

$$
P^{\prime}(x)=-1 / g(x), \quad \Pi^{\prime}(\zeta)=1 / P^{\prime}(\Pi(t))=-g(\Pi(t)) .
$$

Before continuing, let us also record the formulas for $\psi_{x}$ and $\psi_{t}$ :

$$
\psi_{x}=\int_{0}^{T-t} z_{x}(x, t, \tau) d \tau, \quad \psi_{t}=\int_{0}^{T-t} z_{t}(x, t, \tau) d \tau-z(x, t, T-t)
$$

where, with the notation $\hat{y}_{x}:=y_{x}(x, T-t-\tau), \hat{y}_{t}:=y_{t}(x, T-t-\tau)$,

$$
z_{x}(x, t, \tau)=-\phi_{x}(\hat{y}, T-t) \hat{y}_{x} \exp \left(\int_{x}^{\hat{y}} \frac{b(s)}{g(s)}\right)+z(x, t, \tau)\left[\frac{b(\hat{y})}{g(\hat{y})} \hat{y}_{x}-\frac{b(x)}{g(x)}\right],
$$

and

$$
z_{t}(x, t, \tau)=-\phi_{x}(\hat{y}, T-t) \hat{y}_{t} \exp \left(\int_{x}^{\hat{y}} \frac{b(s)}{g(s)}\right)+z(x, t, \tau)\left[\frac{b(\hat{y})}{g(\hat{y})} \hat{y}_{t}\right] .
$$

It is clear that $y \in C\left(\bar{\Pi}_{T}\right)$. Note that because of our assumptions about $g(x)$, both $P(x)$ and $\Pi(t)$ are strictly increasing and vanish when their arguments are zero. In particular, for each $t \in(0, T)$, the set $Z(t):=\{x \mid 0<x<\Pi(t)\}$ is a nonempty open interval, and all of $y, y_{x}$, $y_{t}$ vanish identically for $x \in Z(t)$. With this in mind, it is not hard to see that all of $y, y_{x}, y_{t}$ are continuous and bounded on $\Pi_{T}$. Similarly, for $0<t<T, 0<\tau<T-t, \hat{y}(x, t), \hat{y}_{x}(x, t)$, $\hat{y}_{t}(x, t)$ all vanish identically for $x \in Z(T-t-\tau)$, and thus all of $\hat{y}, \hat{y}_{x}, \hat{y}_{t}$ are bounded on $\Pi_{T}$. Since $\operatorname{supp}(\phi) \subseteq \Pi_{T}$, this in turn implies that $z(x, t, \tau), z_{x}(x, t, \tau), z_{t}(x, t, \tau)$ also vanish for $x \in Z(T-t-\tau)$. Using the formulas (5.5), (5.6), along with the observations above, we may easily check that $\psi(0, t)=\psi(x, T)=0$, and $\psi \in C\left(\bar{\Pi}_{T}\right)$.

To show that $\psi \in \mathcal{D}$, it remains to verify that $\psi_{x} \in L^{1}\left(\Pi_{T}\right), \psi_{t} \in L^{\infty}\left(\Pi_{T}\right)$. In view of formula (5.13), showing that $\psi_{t} \in L^{\infty}\left(\Pi_{T}\right)$ boils down to recalling that $\hat{y}_{x}$ and $\hat{y}_{t}$ are bounded, and verifying that we have control of the second term of (5.13). Since $z_{t}$ vanishes on $Z(T-t-\tau)$, we are only concerned with $x \notin Z(T-t-\tau)$. Both $z$ and $b$ are bounded, and for $x \notin Z(T-t-\tau),(5.8),(5.9)$ reveal that $\hat{y}_{t} / g(\hat{y})=1$. 
To show that $\psi_{x} \in L^{1}\left(\Pi_{T}\right)$ we proceed in a similar manner. This time we find that there is a factor of $1 / g(x)$ multiplying a bounded quantity, giving us an $L^{1}$ bound (since $1 / g \in L^{1}$ ), but not an $L^{\infty}$ bound.

REMARK 5.2. In the proof above we have used the duality relationship between the conservation law

$$
\frac{\partial u}{\partial t}+\frac{\partial}{\partial x}(g(x) u)=b(x) u
$$

and the transport equation (5.4). With this approach, one proves uniqueness for the (forward in time) original problem by proving existence for the (backward in time) dual problem. Note that we are not claiming uniqueness for solutions of the dual problem (5.4). Indeed reference [24], where this duality relationship is explained more completely, points out that in general, when uniqueness holds for the forward original problem, uniqueness fails for the backward dual problem.

\section{Appendix B. Convergence proof for the first-order difference scheme}

In this section we verify that our first order scheme (3.12) produces approximations to the initial boundary problem (3.1), (3.3), (3.4) that converge (along a subsequence) to a weak solution in the sense of Definition 5.1. There are two factors that complicate our analysis. First, solutions are not necessarily bounded, due to the fact that the coefficient vanishes at $x=0$. Second, the source terms create discontinuities in the solution, and more importantly, lead to increases in the spatial variation of the solution. When analyzing first order schemes for simpler conservation laws, it is standard practice to proceed by proving a bound on the amplitude of the approximate solutions, along with a bound on the spatial variation, and a time-continuity estimate. By standard results [25], one can then verify convergence of the approximations along a subsequence as the mesh size tends to zero. Since bounds on the amplitude and the spatial variation are not possible in the present situation, we will instead establish these bounds for the numerical flux approximations defined by (6.1) below. These two bounds, along with a time continuity estimate, allow us to prove that the numerical flux converges, and then we can recover the actual solution $u$, since the value of the flux $g(x) u+\gamma(t) a(x)$ uniquely determines the value of $u$ for this linear problem.

Denote the numerical flux by

$$
h_{j}^{n}:=g_{j} U_{j}^{n}+\gamma^{n} a_{j},
$$

and let

$$
u^{\Delta}(x, t):=\sum_{j=0}^{J^{\Delta}} \sum_{n=0}^{N^{\Delta}} \chi_{j}^{n}(x, t) U_{j}^{n}, \quad h^{\Delta}(x, t):=\sum_{j=0}^{J^{\Delta}} \sum_{n=0}^{N^{\Delta}} \chi_{j}^{n}(x, t) h_{j}^{n} .
$$

As mentioned above, we will prove compactness for the sequence $h^{\Delta}$, and then recover the solution $u$ by inverting $h$. We proceed via a sequence of lemmas which lead up to our main convergence result, Theorem 6.1 . 
LEMMA 6.1. If the CFL condition (3.11) is satisfied, the numerical solution is nonnegative and the numerical flux is nonpositive, i.e.,

$$
U_{j}^{n} \geq 0, \quad h_{j}^{n} \leq 0 \quad \text { for } j=1, \ldots, J^{\Delta}, n=1, \ldots, N^{\Delta} .
$$

Proof. For the first part of (6.2), assume that $U_{j}^{n} \geq 0$. Then the right-hand side of the marching formula

$$
U_{j}^{n+1}=\left(1+\lambda g_{j}+\Delta t b_{j}^{n}\right) U_{j}^{n}-\lambda g_{j+1} U_{j+1}^{n}-\lambda \gamma^{n}\left(a_{j+1}-a_{j}\right)
$$

is also nonnegative due to the CFL condition and properties (3.5) and (3.6), respectively. It is then clear that $U_{j}^{n+1} \geq 0$, and the desired result follows by induction on $n$. For the second part of (6.2), we check (6.1) and use the following facts: $U_{j}^{n} \geq 0, g_{j} \leq 0, \gamma^{n} \leq 0, a_{j} \geq 0$.

The following lemma provides the discrete version of the bound (5.1) on the amplitude of the flux that is satisfied by a weak solution to the initial value problem (3.1), (3.3), (3.4).

LEMMA 6.2. The numerical flux $h_{j}^{n}$ is bounded independently of $\Delta$, i.e.,

$$
\left|h_{j}^{n}\right| \leq C_{1} \text { for all } 0 \leq j \leq J^{\Delta} \text { and } 0 \leq n \leq N_{\Delta} .
$$

Proof. It is easily checked that the flux evolves according to

$$
h_{j}^{n+1}=\left(1+\lambda g_{j}\right) h_{j}^{n}-\lambda g_{j+1} h_{j+1}^{n}+\Delta t g_{j} b_{j}^{n} U_{j}^{n}+a_{j}\left(\gamma^{n+1}-\gamma^{n}\right) .
$$

From this expression, along with the observation that $\Delta t g_{j} b_{j}^{n} U_{j}^{n} \geq 0$, we obtain

$$
h_{j}^{n+1} \geq\left(1+\lambda g_{j}\right) h_{j}^{n}-\lambda g_{j+1} h_{j+1}^{n}+a_{j}\left(\gamma^{n+1}-\gamma^{n}\right) \geq \min \left\{h_{j}^{n}, h_{j+1}^{n}\right\}+a_{j}\left(\gamma^{n+1}-\gamma^{n}\right) .
$$

Recalling that $h_{j}^{n} \leq 0$, we now see that

$$
\left|h_{j}^{n}\right| \leq \max _{j}\left|h_{j}^{0}\right|+2 A \Gamma \leq G\left\|u_{0}\right\|+2 A \Gamma .
$$

LEMMA 6.3. There is a constant $C_{2}$ independent of the mesh size $\Delta$ such that the following spatial variation bound for the numerical flux holds:

$$
\sum_{j=1}^{J^{\Delta}}\left|\Delta_{-} h_{j}^{n}\right| \leq C_{2}, \quad 0 \leq n \leq N^{\Delta} .
$$

Proof. Starting from the expression (6.4) for the evolution of the numerical flux, we find that the flux differences evolve according to

$$
\begin{aligned}
\Delta_{-} h_{j}^{n+1}= & \left(1-\lambda g_{j-1}\right) \Delta_{+} h_{j-1}^{n}-\lambda g_{j} \Delta_{+} h_{j}^{n}+b_{j}^{n} \Delta_{t} \Delta_{-} h_{j}^{n} \\
& +h_{j-1} \Delta t \Delta_{-} b_{j}^{n}+\left(\gamma^{n+1}-\gamma^{n}\right) \Delta_{-} a_{j}-\Delta t \gamma^{n} b_{j}^{n} \Delta_{-} a_{j}-\Delta t \gamma^{n} a_{j-1} \Delta_{-} b_{j}^{n} .
\end{aligned}
$$

Taking absolute values and applying the CFL condition (3.11), we get

$$
\begin{aligned}
\sum_{j=1}^{J^{\Delta}}\left|\Delta_{-} h_{j}^{n+1}\right| \leq & (1+B \Delta t) \sum_{j=1}^{J^{\Delta}}\left|\Delta_{-} h_{j}^{n}\right|+\|h\| \Delta t \sum_{j=1}^{J^{\Delta}}\left|\Delta_{-} b_{j}^{n}\right| \\
& +\left|\gamma^{n+1}-\gamma^{n}\right||a|_{B V}+\Delta t \Gamma B|a|_{B V}+\Delta t \Gamma A \sum_{j=1}^{J^{\Delta}}\left|\Delta_{-} b_{j}^{n}\right| .
\end{aligned}
$$


Applying this inequality inductively, we arrive at

$$
\begin{aligned}
\sum_{j=1}^{J^{\Delta}}\left|\Delta_{-} h_{j}^{n}\right| \leq & (1+B \Delta t)^{n}\left(\sum_{j=1}^{J^{\Delta}}\left|\Delta_{-} h_{j}^{0}\right|+\|h\| \Delta t \sum_{\nu=0}^{n-1} \sum_{j=1}^{J^{\Delta}}\left|\Delta_{-} b_{j}^{\nu}\right|\right. \\
& \left.+\sum_{\nu=0}^{n-1}\left|\gamma^{\nu+1}-\gamma^{\nu}\right||a|_{B V}+n \Delta t \Gamma B|a|_{B V}+\Delta t \Gamma A \sum_{\nu=0}^{n-1} \sum_{j=1}^{J^{\Delta}}\left|\Delta_{-} b_{j}^{\nu}\right|\right) \\
\leq & \mathrm{e}^{B T}\left(\sum_{j=1}^{J^{\Delta}}\left|\Delta_{-} h_{j}^{0}\right|+\|h\||b|_{B V}+|\gamma|_{B V}|a|_{B V}+\Gamma T B|a|_{B V}+\Gamma A|b|_{B V}\right) .
\end{aligned}
$$

The proof is completed via the estimate

$$
\sum_{j=1}^{J^{\Delta}}\left|\Delta_{-} h_{j}^{0}\right| \leq G\left|u_{0}\right|_{B V}+\| u_{0}|||g|_{B V}+\Gamma|a|_{B V} .
$$

Here $|b|_{B V}$ denotes two-dimensional variation, while $|a|_{B V},|\gamma|_{B V}$ and $|g|_{B V}$ denote onedimensional variation.

LEMMA 6.4. The $L^{1}$ norm of the approximate solution is bounded for $t \in[0, T]$, i.e., there is a constant $C_{3}$ independent of the mesh size $\Delta$ such that

$$
\sum_{j=0}^{J^{\Delta}}\left|U_{j}^{n}\right| \Delta x \leq C_{3}, \quad 0 \leq n \leq N^{\Delta} .
$$

Proof. From the definition of the scheme (3.12),

$$
\left|U_{j}^{n+1}\right| \leq\left|U_{j}^{n}\right|+\lambda\left|\Delta_{+} h_{j}^{n}\right|+\Delta t B\left|U_{j}^{n}\right| .
$$

Multiplying by $\Delta x$ and summing over $j$ yields

$$
\sum_{j=0}^{J^{\Delta}}\left|U_{j}^{n+1}\right| \Delta x \leq(1+B \Delta t) \sum_{j=0}^{J^{\Delta}}\left|U_{j}^{n}\right| \Delta x+\lambda C_{2} \Delta x .
$$

Using this inequality to proceed inductively, and applying $\lambda \Delta x=\Delta t$, we get

$$
\sum_{j=0}^{J^{\Delta}}\left|U_{j}^{n}\right| \Delta x \leq \mathrm{e}^{B T} \sum_{j=0}^{J^{\Delta}}\left|U_{j}^{0}\right| \Delta x+C_{2} \frac{\mathrm{e}^{B T}-1}{B} .
$$

LEMMA 6.5. There is a constant $C_{4}$ independent of the mesh size $\Delta$ such that

$$
\sum_{j=0}^{J^{\Delta}}\left|U_{j}^{n+1}-U_{j}^{n}\right| \Delta x \leq C_{4} \Delta t, \quad 0 \leq n \leq N^{\Delta}-1 .
$$

Proof. From the definition of the scheme, and using $\lambda \Delta x=\Delta t$, we have

$$
\sum_{j=0}^{J^{\Delta}}\left|U_{j}^{n+1}-U_{j}^{n}\right| \Delta x \leq \lambda \sum_{j=1}^{J^{\Delta}}\left|\Delta_{-} h_{j}^{n}\right| \Delta x+B \Delta t \sum_{j=0}^{J^{\Delta}}\left|U_{j}^{n}\right| \Delta x \leq C_{2} \Delta t+B C_{3} \Delta t .
$$


THEOREM 6.1. The approximations $u^{\Delta}$ converge along a subsequence a.e. in $\Pi_{T}$ to a weak solution $u$ of the initial value problem (3.1), (3.3), (3.4). For each fixed $t \in[0, T]$, as $\Delta \rightarrow 0$ along this subsequence,

$$
u^{\Delta}(\cdot, t) \longrightarrow u(\cdot, t) \text { in } L_{g}^{1}([0, X]) .
$$

Additionally, for each $\epsilon \in(0, X), u^{\Delta}(\cdot, t)$ converges to $u(\cdot, t)$ along the same subsequence in $L^{1}([\epsilon, X] \times[0, T])$ as $\Delta \rightarrow 0$. If the sieve term is time-independent, i.e., $b=b(x)$, and $1 / g \in L^{1}[0, X]$, then the entire sequence converges to the unique weak solution guaranteed by Theorem 5.1.

Proof. Let $S=\left\{t_{1}, t_{2}, \ldots\right\}$ be a countable dense subset of $[0, T]$. For the sequence of functions $h^{\Delta}\left(\cdot, t_{1}\right)$ we have an $L^{\infty}$ bound (Lemma 6.2), and a bound on the total variation (Lemma 6.3). Also, the $L^{1}$ bound for $u^{\Delta}\left(\cdot, t_{1}\right)$ (Lemma 6.4) readily implies an $L^{1}$ bound for $h^{\Delta}$. All of these bounds are independent of the mesh size $\Delta$. By standard compactness results, there is a function $h^{\lim }\left(\cdot, t_{1}\right) \in L^{1}([0, X]) \cap L^{\infty}([0, X])$ and a subsequence of $\left\{h^{\Delta}\left(\cdot, t_{1}\right)\right\}_{\Delta}$, which we do not bother to relabel, such that $h^{\Delta}\left(\cdot, t_{1}\right) \rightarrow h^{\lim }\left(\cdot, t_{1}\right)$ in $L^{1}([0, X])$ and a.e. in $[0, X]$. In view of the identity

$$
u^{\Delta}\left(x, t_{1}\right)=\frac{h^{\Delta}\left(x, t_{1}\right)-\gamma^{\Delta}(t) a^{\Delta}(x)}{g^{\Delta}(x)}, \quad x \in(0, X]
$$

we recover the solution $u$ from $h^{\lim }$ via

$$
u\left(x, t_{1}\right):=\frac{h^{\lim }\left(x, t_{1}\right)-\gamma\left(t_{1}\right) a(x)}{g(x)}, \quad x \in(0, X] .
$$

It is clear that the right-hand side of (6.10) converges to the right-hand side of (6.11) a.e. in $[0, X]$, and thus $u^{\Delta}\left(\cdot, t_{1}\right) \rightarrow u\left(\cdot, t_{1}\right)$ a.e. in $[0, X]$. Next, we claim that

$$
\int_{0}^{X}\left|u^{\Delta}\left(x, t_{1}\right)-u\left(x, t_{1}\right)\right||g(x)| d x \longrightarrow 0 \text { as } \Delta \rightarrow 0 .
$$

To prove this claim, we use the triangle inequality to derive

$$
\begin{aligned}
\int_{0}^{X}\left|u^{\Delta}\left(x, t_{1}\right)-u\left(x, t_{1}\right)\right||g(x)| d x \leq & \int_{0}^{X}\left|g^{\Delta}(x) u^{\Delta}\left(x, t_{1}\right)-g(x) u\left(x, t_{1}\right)\right| d x \\
& +\int_{0}^{X}\left|u^{\Delta}\left(x, t_{1}\right)\right|\left|g(x)-g^{\Delta}(x)\right| d x .
\end{aligned}
$$

The first integral on the right-hand side of (6.13) converges to zero; this follows from $h^{\Delta}\left(\cdot, t_{1}\right) \rightarrow$ $h\left(\cdot, t_{1}\right)$ in $L^{1}([0, X])$. The second integral can be estimated by

$$
\int_{0}^{X}\left|u^{\Delta}\left(x, t_{1}\right)\right|\left|g(x)-g^{\Delta}(x)\right| d x \leq \sup _{x \in[0, X]}\left|g(x)-g^{\Delta}(x)\right| \int_{0}^{X}\left|u^{\Delta}\left(x, t_{1}\right)\right| d x .
$$

The term on the right side of (6.14) converges to zero, thanks to our uniform $L^{1}$ bound on $u^{\Delta}$ (Lemma 6.4), and the fact that $g$ is uniformly continuous on $[0, X]$.

We can repeat this argument with $t_{2}$ replacing $t_{1}$, and starting from a subsequence of the subsequence used to construct $u\left(\cdot, t_{1}\right)$, giving us $u\left(\cdot, t_{2}\right) \in L_{g}^{1}([0, X])$ and $u^{\Delta}\left(\cdot, t_{2}\right) \rightarrow u\left(\cdot, t_{2}\right)$. Continuing this way, taking a subsequence of the previous one at each step, and then using 
the standard diagonalization process, we get a function $u(x, t)$, which is defined for $t \in S$ such that $u(\cdot, t) \in L_{g}^{1}\left([0, X]\right.$, and $u^{\Delta}(\cdot, t) \rightarrow u(\cdot, t)$ in $L_{g}^{1}([0, X])$ for $t \in S$.

As a consequence of the time continuity estimate (Lemma 6.5), $t \mapsto u(\cdot, t)$ is a uniformly continuous mapping of the dense subset $S$ into $L_{g}^{1}([0, X])$. Since $L_{g}^{1}([0, X])$ is complete, the mapping can be extended uniquely to a uniformly continuous mapping $t \mapsto u(\cdot, t)$ whose domain is the entire interval $[0, T]$. A straightforward estimate using the triangle inequality and uniform continuity in time shows that $u^{\Delta}(\cdot, t) \rightarrow u(\cdot, t)$ in $L_{g}^{1}([0, X])$ for $t \in[0, T]$.

For any $\epsilon>0$, both $u^{\Delta}(\cdot, t)$ and $u(\cdot, t)$ are bounded in $[\epsilon, X]$, and the bound is independent of both $\Delta$ and $t \in[0, T]$. The quantity

$$
\Lambda^{\Delta}(t):=\int_{\epsilon}^{X}\left|u^{\Delta}(x, t)-u(x, t)\right| d x
$$

thus converges boundedly to zero, and so by the bounded convergence theorem,

$$
\int_{0}^{T} \int_{\epsilon}^{X}\left|u^{\Delta}(x, t)-u(x, t)\right| d x d t=\int_{0}^{T} \Lambda^{\Delta}(t) d t
$$

also converges to zero. By passing to a further subsequence, we have $u^{\Delta} \rightarrow u$ boundedly a.e. in $[\epsilon, X] \times[0, T]$. By taking a countable decreasing sequence $\left\{\epsilon_{i}\right\}_{i=1}^{\infty}$ with $\epsilon_{i} \rightarrow 0$, and employing another diagonalization process, we can arrange that the subsequence of approximations $u^{\Delta}$ converges to $u$ a.e. in $\Pi_{T}$.

In what follows, we will use the observations that $u(\cdot, t) \in L^{1}([0, X])$, and $u \in L^{1}\left(\Pi_{T}\right)$. These facts follow easily from Lemma 6.4, along with an application of Fatou's Lemma. Also, since $g(x)$ in bounded away from zero if $x$ is bounded away from zero, for any $\epsilon \in(0, X)$, the solution $u$ lies in $C\left([0, T] ; L^{1}([\epsilon, X])\right)$. Moreover, for the subsequence constructed above,

$$
u^{\Delta}(\cdot, t) \longrightarrow u \text { in } L^{1}([\epsilon, X]) \text { and boundedly a.e. in }[\epsilon, X], \quad t \in[0, T] .
$$

Our goal now is to show that $u$ is a weak solution. We start with the observation that the bound (5.1) on the amplitude of the flux is satisfied for the limit solution $u$; this is a direct result of Lemma 6.2. To show that (5.2) is satisfied, fix $\varepsilon \in(0, X)$, and let $\phi \in C^{1}([\varepsilon, X] \times[0, T])$ with support contained in $[\varepsilon, X] \times[0, T)$. We write the scheme in the form

$$
\frac{U_{j}^{n+1}-U_{j}^{n}}{\Delta t}+\frac{\Delta_{+}}{\Delta x}\left(g_{j} U_{j}^{n}+\gamma^{n} a_{j}\right)-b_{j}^{n} U_{j}^{n}=0,
$$

multiply by $\phi\left(x_{j}, t^{n}\right) \Delta t \Delta x$, and then sum over $0 \leq n \leq N^{\Delta}-1,0 \leq J^{\Delta}-1$. After two summations by parts (one in $j$, and one in $n$ ), and letting $\phi_{j}^{n}=\phi\left(x_{j}, t^{n}\right)$, we arrive at

$$
\begin{aligned}
& \Delta t \Delta x \sum_{n=0}^{N^{\Delta}-1} \sum_{j=0}^{J^{\Delta}-1} U_{j}^{n} \frac{\phi_{j}^{n}-\phi_{j}^{n-1}}{\Delta t}-\Delta x \sum_{j=0}^{J^{\Delta}-1} U_{j}^{N^{\Delta}} \phi_{j}^{N^{\Delta}-1}+\Delta x \sum_{j=0}^{J^{\Delta}-1} U_{j}^{0} \phi_{j}^{0} \\
& +\Delta t \Delta x \sum_{n=0}^{N^{\Delta}-1} \sum_{j=0}^{J_{-}-1}\left(g_{j} U_{j}^{n}+\gamma^{n} a_{j}\right) \frac{\phi_{j}^{n}-\phi_{j-1}^{n}}{\Delta x}-\Delta t \sum_{n=0}^{N^{\Delta}-1} \gamma^{n} a_{J} \phi_{J}^{n}-1 \\
& +\Delta t \Delta x \sum_{n=0}^{N^{\Delta}-1} \sum_{j=0}^{J_{-} \Delta_{-}} b_{j}^{n} U_{j}^{n} \phi_{j}^{n}=0 .
\end{aligned}
$$


Here we have used $\phi_{0}^{n}=0$ and $U_{J \Delta}^{n}=0$ to eliminate two boundary sums that would otherwise appear. Recalling our bound on $g_{j} U_{j}^{n}+\gamma^{n} a_{j}$, we obtain by the bounded convergence theorem

$$
\Delta t \Delta x \sum_{n=0}^{N^{\Delta}-1} \sum_{j=0}^{J^{\Delta}-1}\left(g_{j} U_{j}^{n}+\gamma^{n} a_{j}\right) \frac{\phi_{j}^{n}-\phi_{j-1}^{n}}{\Delta x} \longrightarrow \int_{0}^{T} \int_{0}^{X}(g(x) u+\gamma(t) a(x)) \frac{\partial \phi}{\partial x}(x, t) d x d t .
$$

Yet another application of the bounded convergence theorem gives

$$
\Delta t \sum_{n=0}^{N^{\Delta}-1} \gamma^{n} a_{J \Delta} \phi_{J \Delta_{-1}}^{n} \longrightarrow \int_{0}^{T} \gamma(t) a(X) \phi(X, t) d t
$$

For the third sum on the first line of (6.16), the bounded convergence theorem gives

$$
\Delta x \sum_{j=0}^{J^{\Delta}-1} U_{j}^{0} \phi_{j}^{0} \longrightarrow \int_{0}^{X} u_{0}(x) \phi(x) d x
$$

For the sum on the last line of (6.16), we use that $u^{\Delta} \rightarrow u$ in $L^{1}([\epsilon, X] \times[0, T])$, along with the assumption that $\phi$ vanishes for $x \leq \epsilon$ to conclude that

$$
\Delta t \Delta x \sum_{n=0}^{N^{\Delta}-1} \sum_{j=0}^{J^{\Delta}-1} b_{j}^{n} U_{j}^{n} \phi_{j}^{n} \longrightarrow \int_{0}^{T} \int_{0}^{X} b(x, t) u \phi d x d t
$$

Similarly, using 6.15 , along with the fact that $\phi(x, T)=0$, we have

$$
\Delta x \sum_{j=0}^{J^{\Delta}-1} U_{j}^{N^{\Delta}} \phi_{j}^{N^{\Delta}-1} \longrightarrow \int_{0}^{X} u(x, T) \phi(x, T) d x=0 .
$$

Finally, for the first sum on the first line of (6.16), using the mean value theorem, we write

$$
U_{j}^{n} \frac{\phi_{j}^{n}-\phi_{j}^{n-1}}{\Delta t}=U_{j}^{n} \frac{\partial \phi}{\partial t}\left(x_{j}, \tau^{n}\right)
$$

where $\left|\tau^{n}-t^{n}\right| \leq \Delta t$. Again using the fact that $u^{\Delta} \rightarrow u$ in $L^{1}([\epsilon, X] \times[0, T])$, along with the assumption that $\phi$ vanishes for $x \leq \epsilon$, we have

$$
\Delta t \Delta x \sum_{n=0}^{N^{\Delta}-1} \sum_{j=0}^{J^{\Delta}-1} U_{j}^{n} \frac{\phi_{j}^{n}-\phi_{j}^{n-1}}{\Delta t} \longrightarrow \int_{0}^{T} \int_{0}^{X} u \frac{\partial \phi}{\partial t} d x d t .
$$

Combining (6.17) through (6.22), we see that for our class of regularized test functions, $u$ satisfies (5.2). We still must show that $u$ satisfies (5.2) for all $\phi \in \mathcal{D}$. Let $\phi \in \mathcal{D}$. For $\epsilon \in(0, X)$, it is possible to construct a set of regularized approximations $\phi^{\epsilon} \in C^{1}([\epsilon, X] \times[0, T])$ with support in $[\epsilon, X] \times[0, T)$ such that

$$
\phi^{\epsilon} \longrightarrow \phi, \quad \frac{\partial \phi^{\epsilon}}{\partial t} \longrightarrow \frac{\partial \phi}{\partial t} \text { boundedly a.e. in } \Pi_{T}, \quad \frac{\partial \phi^{\epsilon}}{\partial x} \longrightarrow \frac{\partial \phi}{\partial x} \text { in } L^{1}\left(\Pi_{T}\right) \text {. }
$$


For our regularized test functions, we have

$$
\begin{aligned}
& \int_{0}^{T} \int_{0}^{X}\left\{u \frac{\partial \phi^{\epsilon}}{\partial t}+(g(x) u-\gamma(t) a(x)) \frac{\partial \phi^{\epsilon}}{\partial x}+b(x, t) u \phi^{\epsilon}\right\} d x d t \\
& -\int_{0}^{T} \gamma(t) a(X) \phi^{\epsilon}(X, t) d t+\int_{0}^{X} u_{0}(x) \phi^{\epsilon}(x, 0) d x=0 .
\end{aligned}
$$

Now letting $\epsilon \downarrow 0$, recalling that $u \in L^{1}\left(\Pi_{T}\right), g(x) u-\gamma(t) a(x) \in L^{\infty}\left(\Pi_{T}\right)$, and applying the dominated convergence theorem yields (5.2) for $\psi \in \mathcal{D}$.

When $b=b(x), 1 / g \in L^{1}([0, X])$, we have uniqueness (Theorem 5.1), and so the entire sequence converges to to the unique weak solution $u$.

\section{Acknowledgements}

We acknowledge support by the Collaborative Research Center (Sonderforschungsbereich) 404 at the University of Stuttgart, the BeMatA program of the Research Council of Norway, and the European network HYKE, funded by the EC as contract HPRN-CT-2002-00282.

\section{References}

1. H.M. Hulburt and S. Katz, Some problems in particle technology. Chem. Eng. Sci. 19 (1964) 555-574.

2. D. Verkoeijen, G.A. Pouw, G.M.H. Meesters and B. Scarlett, Population balances for particulate processes - a volume approach. Chem. Eng. Sci. 57 (2002) 2287-2303.

3. A.D. Randolph and M.A. Larson, Transient and steady state size distributions in continuous mixed suspension crystallizers. AIChE J. 8 (1962) 639-645.

4. K.J. Valentas, O. Bilous and N.R. Amundson, Analysis of breakage in dispersed phase systems. Ind. Eng. Chem. Fund. 5 (1966) 271-279.

5. K.J. Valentas and N.R. Amundson, Breakage and coalescence in dispersed phase systems. Ind. Eng. Chem. Fund. 5 (1966) 533-542.

6. A.G. Frederickson, D. Ramkrishna and H.M. Tsuchiya, Statistics and dynamics of procaryotic cell populations. Math. Biosci. 1 (1967) 327-347.

7. K.V.S. Sastry and D.W. Fuerstenau, Size distribution of agglomerates in coalescing dispersed phase systems. Ind. Eng. Chem. Fund. 9 (1970) 145-149.

8. D. Ramkrishna, Population Balances - Theories and Applications to Particulate Systems in Engineering, San Diego: Academic Press (2000) 371 pp.

9. B.J. McCoy, A population balance framework for nucleation, growth, and aggregation. Chem. Eng. Sci. 57 (2002) 2279-2285.

10. M.M. Attarakih, H.-J. Bart and N.M. Faqir, Numerical solution of the spatially distributed population balance equation describing the hydrodynamics of interacting liquid-liquid dispersions. Chem. Eng. Sci. 59 (2004) 2567-2592.

11. R.H. Perry, D.W. Green and J.O. Maloney, Perry's Chemical Engineer's Handbook, Seventh Ed. New York: McGraw-Hill (1998) 2640 pp.

12. J.M. Menacho, Comments on 'A contribution to ball wear and ball size distribution in tumbling mills' by L.A. Vermeulen. Powder Technol. 47 (1986) 97-99.

13. J.M. Menacho and F. Concha, Mathematical model of ball wear in grinding mills: I. Zero-order wear rate. Powder Technol. 47 (1986) 87-96.

14. J.M. Menacho and F. Concha, Mathematical model of ball wear in grinding mills: II. General solution. Powder Technol. 52 (1987) 267-277. 
15. L.A. Vermeulen, A contribution to 'Ball wear and ball size distributions in tumbling ball mills'. Powder Technol. 46 (1986) 281-285.

16. A. Datta and R.K. Rajamani, A direct approach of modeling batch grinding in ball mills using population balance principles and impact energy distribution. Int. J. Mineral Process. 64 (2002) 181-200.

17. K. Yildirim and L.G. Austin, The abrasive wear of cylindrical grinding media. Wear 218 (1998) 15-20.

18. L.G. Austin and R.R. Klimpel, Ball wear and ball size distribution in tumbling ball mills. Powder Technol. 41 (1985) 279-286.

19. F.C. Bond, Wear and size distribution of grinding balls. Trans. Amer. Instn. Min. Met. Engrs. 153 (1943) 373-385.

20. F.C. Bond, Grinding ball size selection. Mining Eng. 10 (1958) 592-595.

21. E.W. Davis, Fine crushing in ball-mills. Trans. Amer. Instn. Min. Met. Engrs. 61 (1919) 250-296.

22. R.J. LeVeque, Finite Volume Schemes for Hyperbolic Problems. Cambridge: Cambridge University Press (2000) $577 \mathrm{pp}$.

23. R.C. McOwen, Partial Differential Equations: Methods and Applications, Second Ed. Upper Saddle Point: Pearson Education (2003) 464 pp.

24. F. Bouchut and F. James, One-dimensional transport equations with discontinuous coefficients. Nonlin. Anal. Real World Appl. 32 (1998) 891-933.

25. M. G. Crandall and A. Majda, Monotone difference approximations for scalar conservation laws. Math. Comp. 34 (1980) 1-21.

26. A. Harten, On a class of high resolution total-variation-stable finite difference schemes. SIAM J. Numer. Anal. 21 (1984) 1-23.

27. A. Harten, S. Osher, B. Engquist, S. Chakravarthy, Some results on uniformly high order accurate essentially non-oscillatory schemes. Appl. Numer. Math. 2 (1986) 347-377.

28. S. Osher, Convergence of generalized MUSCL schemes. SIAM J. Numer. Anal. 22 (1985) 217-235.

29. P.K. Sweby, High resolution schemes using flux limiters for hyperbolic conservation laws. SIAM J. Numer. Anal. 21 (1984) 995-1011. 